\title{
Macrophages mediate cardioprotective cellular postconditioning in acute myocardial infarction
}

\author{
Geoffrey de Couto, ${ }^{1}$ Weixin Liu, ${ }^{1}$ Eleni Tseliou, ${ }^{1}$ Baiming Sun, ${ }^{1}$ Nupur Makkar, ${ }^{1}$ Hideaki Kanazawa, ${ }^{1,2}$ \\ Moshe Arditi, ${ }^{1}$ and Eduardo Marbán ${ }^{1}$ \\ 'Cedars-Sinai Heart Institute, Los Angeles, California, USA. ²Department of Cardiology, Keio University School of Medicine, Tokyo, Japan.
}

\begin{abstract}
Ischemic injury in the heart induces an inflammatory cascade that both repairs damage and exacerbates scar tissue formation. Cardiosphere-derived cells (CDCs) are a stem-like population that is derived ex vivo from cardiac biopsies; they confer both cardioprotection and regeneration in acute myocardial infarction (MI). While the regenerative effects of CDCs in chronic settings have been studied extensively, little is known about how CDCs confer the cardioprotective process known as cellular postconditioning. Here, we used an in vivo rat model of ischemia/reperfusion (IR) injury-induced MI and in vitro coculture assays to investigate how CDCs protect stressed cardiomyocytes. Compared with control animals, animals that received CDCs 20 minutes after IR had reduced infarct size when measured at $\mathbf{4 8}$ hours. CDCs modified the myocardial leukocyte population after ischemic injury. Specifically, introduction of CDCs reduced the number of CD68+ macrophages, and these CDCs secreted factors that polarized macrophages toward a distinctive cardioprotective phenotype that was not M1 or M2. Systemic depletion of macrophages with clodronate abolished CDC-mediated cardioprotection. Using both in vitro coculture assays and a rat model of adoptive transfer after IR, we determined that CDC-conditioned macrophages attenuated cardiomyocyte apoptosis and reduced infarct size, thereby recapitulating the beneficial effects of CDC therapy. Together, our data indicate that CDCs limit acute injury by polarizing an effector macrophage population within the heart.
\end{abstract}

\section{Introduction}

Cardiac ischemic injury recruits a heterogeneous mixture of both protective and cytotoxic cell types. This inflammatory cascade proceeds through a canonical series of events: first, an influx of neutrophils and macrophages clear necrotic debris after inflammation; later, extracellular matrix is deposited and growth factors are released; and finally, the inflammation is resolved, followed by maturation of the scar $(1,2)$. Thus, inflammation converts necrotic tissue into scar, but the abundance of cytotoxic cells recruited into the myocardium has the potential to exacerbate injury. Despite longstanding appreciation of the detrimental inflammatory consequences of ischemia and reperfusion (3-5), clinically useful interventions targeting this pathway are lacking.

Macrophages are an important, heterogeneous cell population that may be functionally traced to their site of origin (bone marrow $[\mathrm{BM}]$ vs. yolk sac) and spatial localization (tissue resident vs. peripheral, monocyte derived). Resident macrophages confer environmental homeostasis $(6,7)$ and maintain residency through local proliferation (8-10). However, following tissue injury, inflammatory monocytes are recruited to the site of injury, differentiate into macrophages, and proliferate to support repair (11-13). In myocardial infarction (MI) and ischemia/reperfusion (IR) injury, monocytes are recruited from BM and splenic reserves in a biphasic man-

\section{Related Commentary: p. 2927}

Conflict of interest: Eduardo Marbán holds equity in, and serves as unpaid advisor to Capricor Therapeutics Inc.

Submitted: February 4, 2015; Accepted: June 5, 2015.

Reference information: / Clin Invest. 2015;125(8):3147-3162. doi:10.1172/JCI81321. ner (14-16). The early Ly6 $\mathrm{C}^{\text {hi }}$ population, which is associated with the $\mathrm{M} 1$ proinflammatory macrophage phenotype, is recruited as a result of increased MCP-1/CCR2 chemokine/monocyte receptor interaction and elevated expression of endothelial adhesion molecules (17). Between days 4 and 7 after MI, a late Ly $6 C^{\text {lo }}$ population, associated with the M2 "healing" phenotype, infiltrates the myocardium (18). Interestingly, targeted depletion of either population with clodronate liposomes leads to impaired infarct healing (14, 19, 20). Characterization of M1 and M2 subpopulations has been well-established (21-23), and macrophages can assume a multitude of activated states in response to microenvironmental cues (6, 24). In fact, within the adult heart at least 4 distinct resident macrophage subsets exist under steady-state conditions (25). Following MI, endogenous cardiac-derived chemotactic signals and danger-associated molecular patterns are released from the infarcted tissue, promoting the expansion of resident and monocyte-derived macrophages into distinct phenotypes.

Cardiosphere-derived cells (CDCs) (26) are a unique heartderived cell type that confers functional and structural benefits, including reduction of infarct size, improvement of cardiac function, enhanced angiogenesis, and modulation of the inflammatory response after MI (27-31). Recently, in a porcine model, we have found that CDCs are able to confer acute cardioprotection when administered 30 minutes after IR (a phenomenon called "cellular postconditioning"; ref. 32). While this was the first demonstration that infarct size could be reduced by delayed therapy, the mechanism was not addressed. Here, we confirm in a rat model that administration of CDCs following a delay after IR reduces infarct mass and improves function. These effects are abolished by systemic macrophage depletion and reproduced 
A

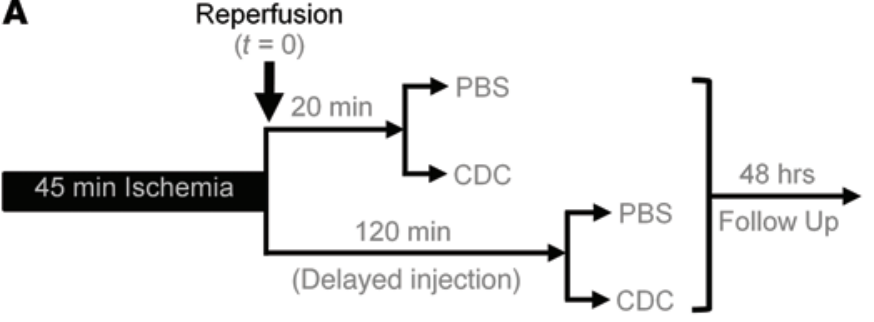

$\mathbf{B}$

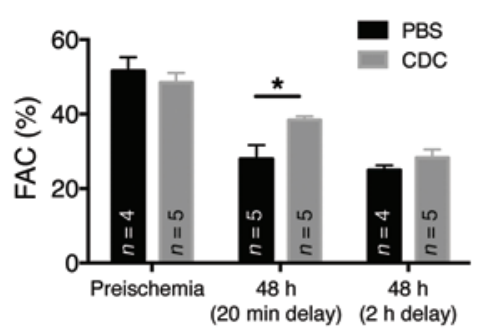

C

임
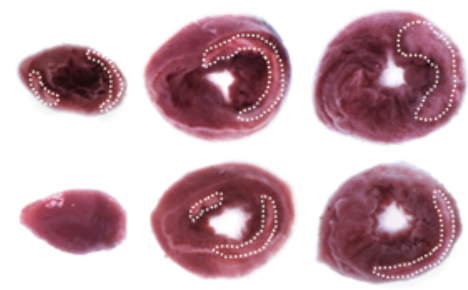

仓ั
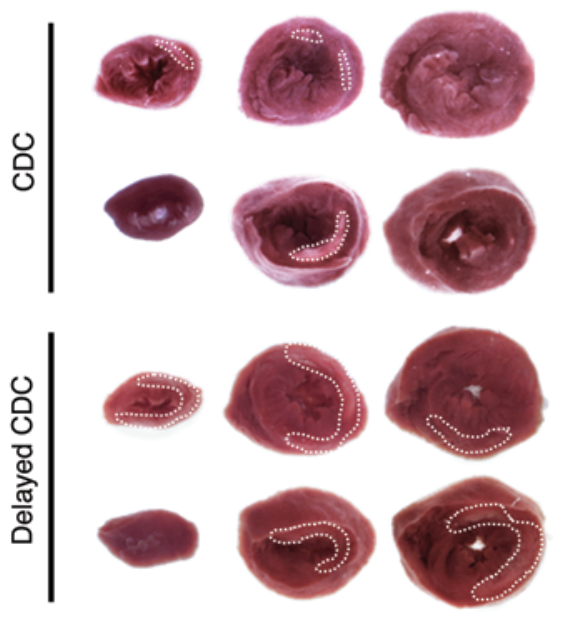

D
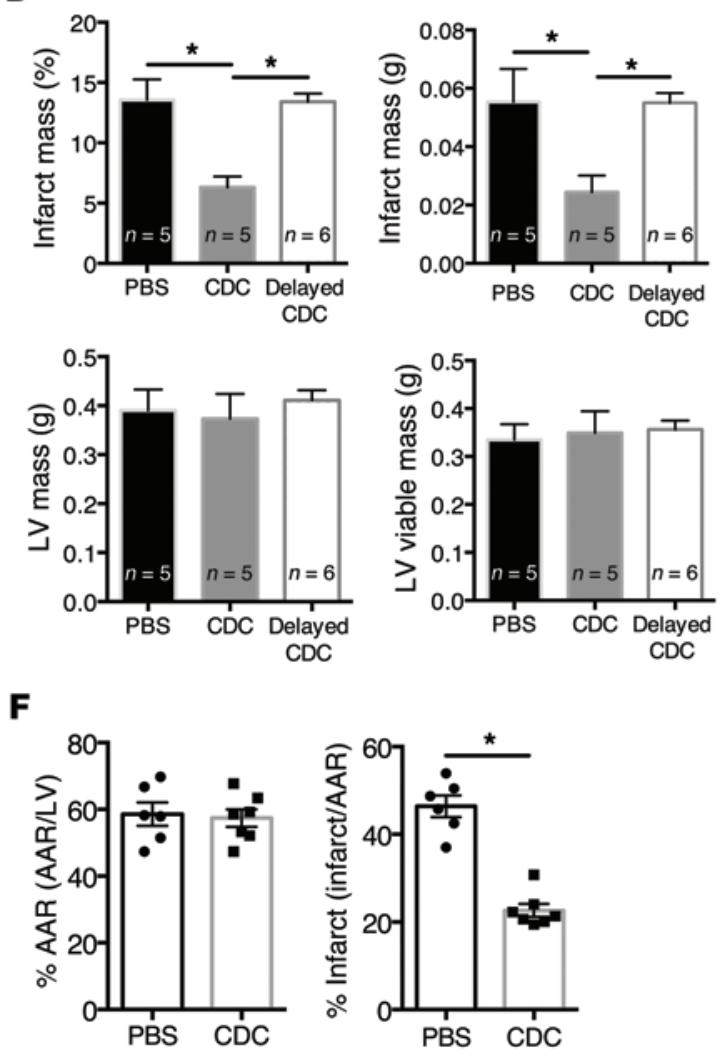
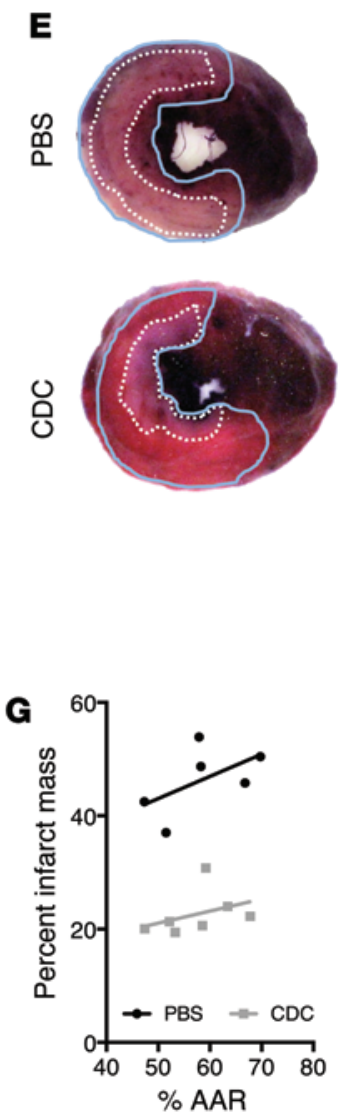

Figure 1. CDCs confer cardioprotection to the ischemic myocardium within $\mathbf{2 0}$ minutes of reperfusion. (A) Schematic of infusion protocol. Rats underwent 45 minutes of ischemia, followed by either 20 minutes or 120 minutes (delayed injection) of reperfusion prior to infusion of CDCs ( $5 \times 10^{5}$ cells per 100 $\mu \mathrm{l})$ or PBS control $(100 \mu \mathrm{l})$ into the LV cavity during aortic cross-clamp. Animals were assessed 48 hours later. (B) Ejection fraction, as measured by echocardiography, was significantly preserved in CDC-treated animals at 48 hours with a 20-minute, but not a 120-minute, delay of infusion ( $n=4-5$ rats per group). FAC, fractional area of change. (C) Representative TTC-stained hearts from animals at 48 hours following IR injury. (D) Quantitative measurements of TTC-stained hearts, depicted as percentage of infarct mass, infarct mass, LV mass, and LV viable mass ( $n=5-6$ rats per group). (E) Representative images of gentian violet- and TTC-stained hearts isolated from rats 48 hours following treatment with PBS or CDCs $\left(5 \times 10^{5}\right)$ and $(\mathbf{F})$ quantitative analysis of the AAR (black line), infarct area (gray line), and LV area of TTC-stained hearts ( $n=6-7$ rats per group). (G) Linear regression of percentage of infarct mass vs. AAR shows no significant interaction when analyzed for homogeneity of regressions $(F=0.23, P=0.64)$ but a difference between adjusted means of infarct mass $(F=84.5, P<0.001)$ by analysis of covariance. Graphs depict mean \pm SEM. Statistical significance was determined using 1 -way ANOVA followed by Bonferroni's multiple comparisons test. ${ }^{*} P<0.05$.

by adoptive transfer of CDC-primed macrophages. Distinctive changes in macrophage gene expression and function underlie the cardioprotective effects of CDCs.

\section{Results}

Allogeneic CDCs confer cardioprotection within 20 minutes of infusion after IR. To investigate the role of CDCs in cellular postconditioning, we designed a protocol that would simulate clinical IR injury. As an urgent priority, patients with MI undergo prompt angioplasty to reopen the occluded coronary artery. After flow has been reestablished, the use of adjunctive therapy can be considered without distraction. Adjunctive cell therapy would require thawing of an allogeneic, off-the-shelf product and preparation for administration, which could introduce a delay of up to 20 minutes. Therefore, in our rat model, we subjected rats to 45 minutes of ischemia, followed by 20 minutes of reperfusion. Cells (or vehicle) were then delivered to the coronary circulation (Figure 1A). To examine whether cell administration could 

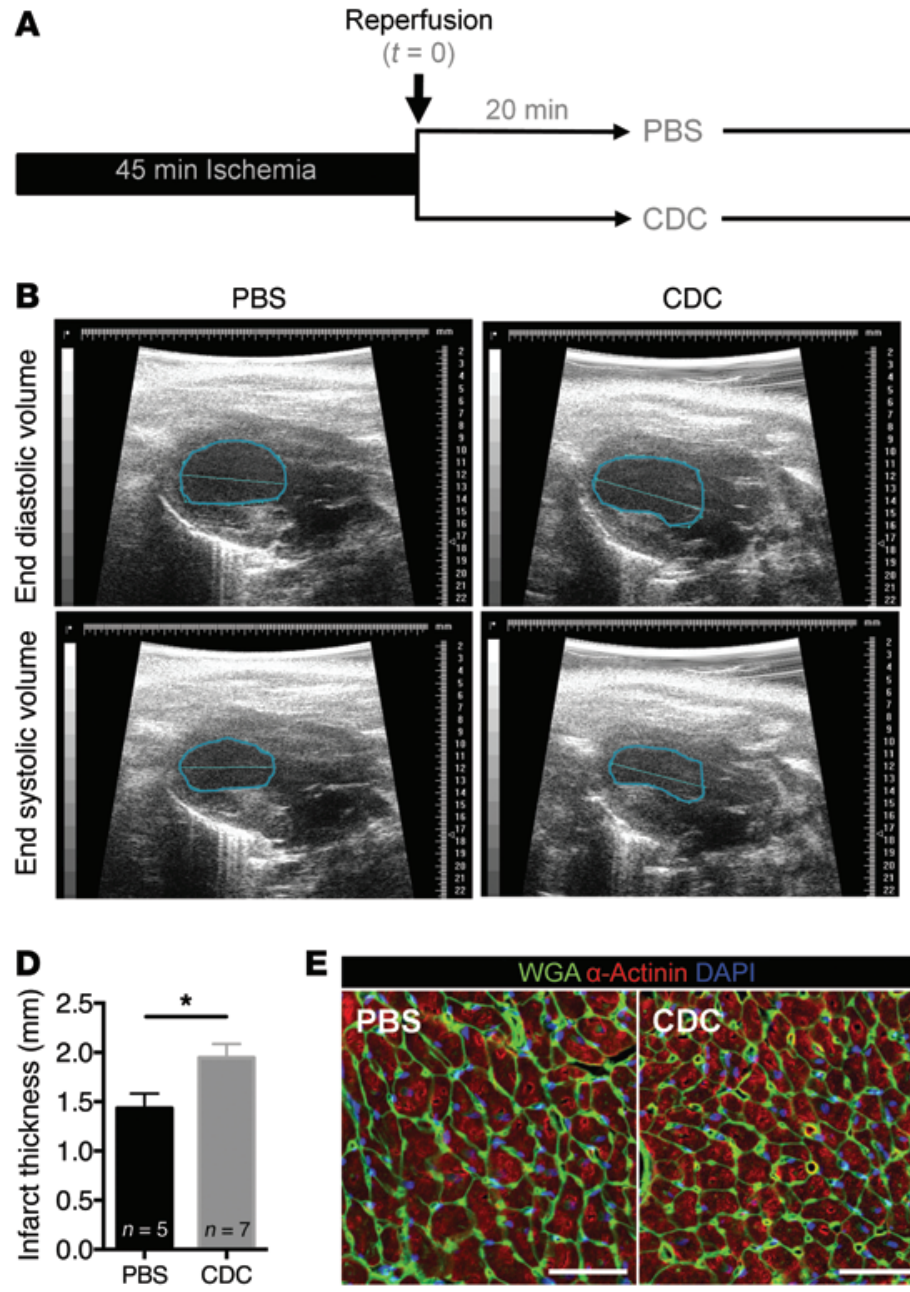

$\mathbf{E}$

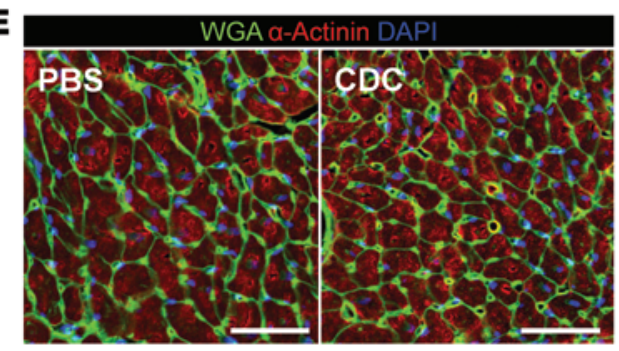

2 week

follow-up
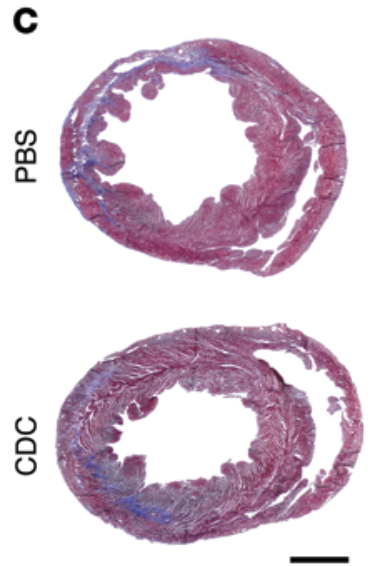

$\mathbf{F}$

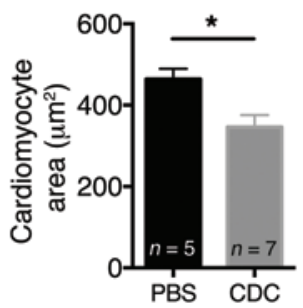

Figure 2. The acute cardioprotective effect of CDCs is sustained for at least 2 weeks following IR. (A) Schematic of infusion protocol. Rats underwent 45 minutes of ischemia, followed by 20 minutes of reperfusion before infusion of CDCs or PBS control. Animals were followed for 2 weeks. (B) Representative echocardiography long-axis images of the LV cavity during diastole and systole from PBS- and CDC-treated animals. (C) Masson's trichrome staining of infarcted hearts from PBS- and CDC-treated animals. Scale bar: $2 \mathrm{~mm}$. (D) Pooled data from Masson's trichrome-stained hearts in C reveal less infarct thinning in CDC-treated animals ( $n=4-5$ rats per group). (E) Immunohistochemical staining of cardiomyocytes in the contralateral infarct zone. Cell size was determined from cardiomyocytes ( $\alpha$-actinin plus wheat-germ agglutinin [WGA]) with centrally localized nuclei (DAPI). Scale bar: $50 \mu \mathrm{m}$. (F) Pooled data from analyses in $\mathbf{E}$ depicting a reduction in cardiomyocyte size in CDC-treated animals ( $n=5-7$ rats per group). Graphs depict mean \pm SEM. Statistical significance was determined using Student's $t$ test and 2-way ANOVA followed by Bonferroni's multiple comparisons test. ${ }^{*} P<0.05$.

be delayed further, we compared the results to those from a "delayed infusion" group, in which CDCs were infused 2 hours after IR (Figure 1A). In general, to enable study of the cardioprotective effect in isolation, we quantified endpoints at 48 hours, well before the regenerative mechanisms of cardiomyocyte proliferation and activation of endogenous cardioblasts come into play (on a time scale of weeks; ref. 29, 33, 34).

CDC-treated animals exhibited preserved cardiac function (Figure 1B) and reduced infarct size (Figure 1, C and D), relative to those of animals treated with vehicle (PBS) control or delayed infusion. We measured area at risk (AAR) and found it to be consistent among animals and independent of the cardioprotective effect (Figure 1, E-G). While these benefits occurred during the acute reparative phase, the functional and structural benefits of CDCs persisted for at least 2 weeks (Figure 2). During this chronic repair phase, cardiac function did not deteriorate, as it did in controls, leading to preserved left ventricular (LV) systolic and diastolic dimensions (Figure 2B and Supplemental Table 1; supplemental material available online with this article; doi:10.1172/JCI81321DS1), less thinning of the LV anterior wall (Figure 2, C and D), attenuated fibrosis (Supplemental Figure 1, $\mathrm{A}$ and $\mathrm{B})$, and reduced hypertrophy of surviving cardiomyocytes (Figure 2, E and F). Thus, CDCs acutely administered after MI reduce myocardial injury at 48 hours, leading to sustained functional and structural benefits.

Infusion of CDCs reduces cardiac stress, attenuating cardiomyocyte death and proinflammatory cytokine expression. The observed reduction in infarct mass may reflect, at least partially, a reduction in programmed cardiomyocyte death. To test this hypothesis, we probed cell death in the infarct, border, and normal zones at various times (Figure $3 \mathrm{~A}$ ) and observed a reduction in cleaved caspase-3 and RIP1 proteins within the infarct (Figure 3, B and C). 
A
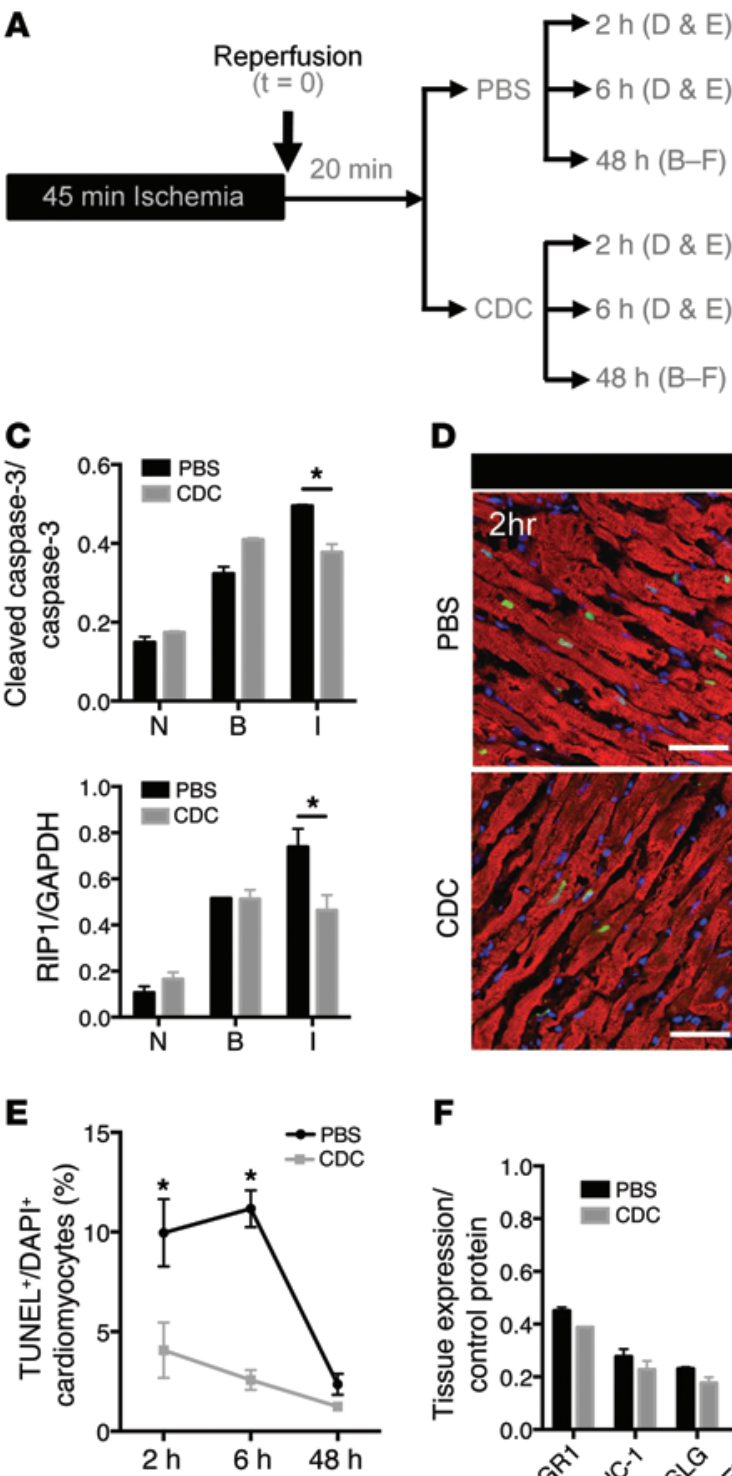

D

$\mathbf{F}$
B
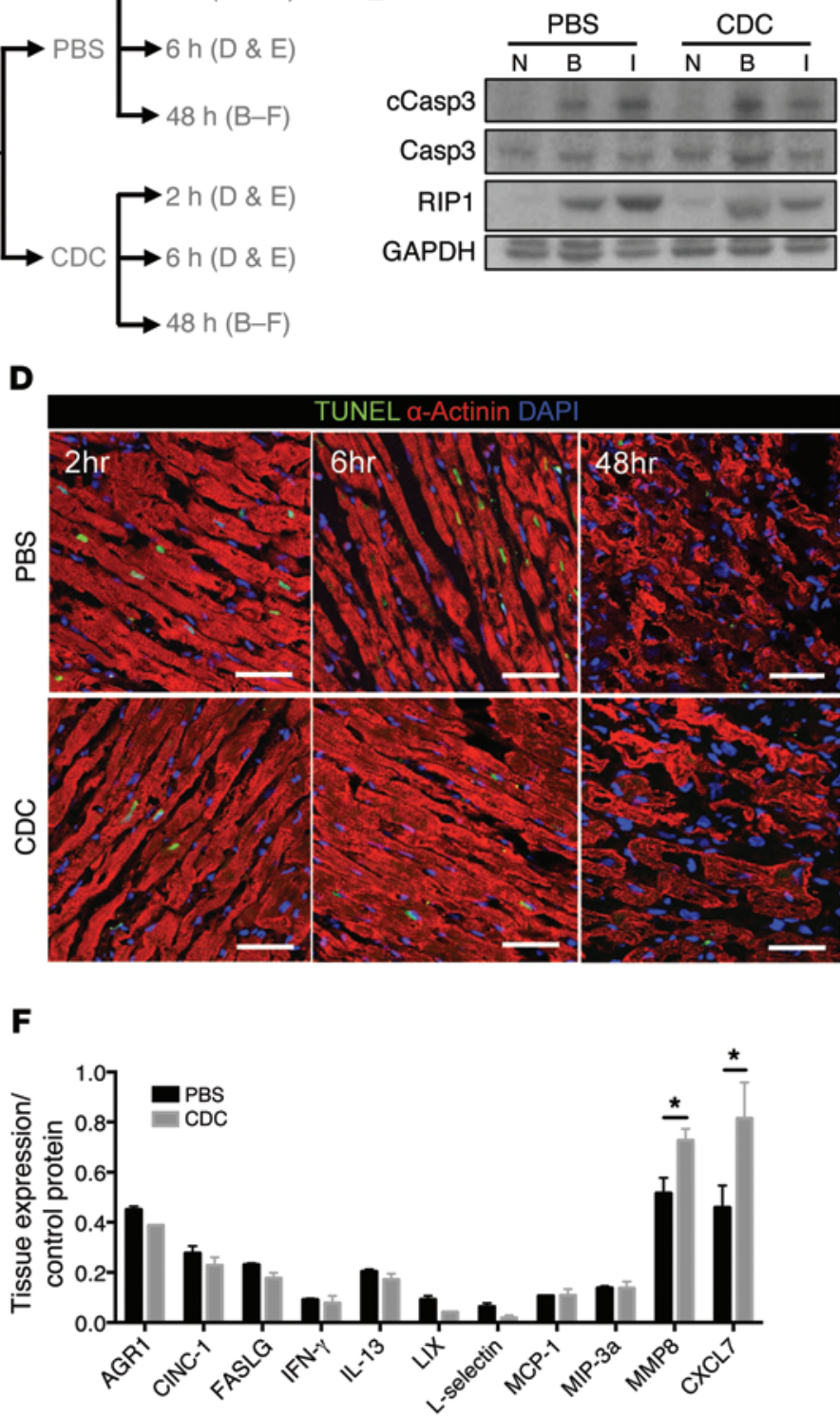

Figure 3. Infusion of CDCs after IR reduces cardiomyocyte death and alters tissue proinflammatory cytokine expression. (A) Schematic of infusion and tissue harvest protocol. Animals underwent 45 minutes of ischemia, followed by 20 minutes of reperfusion prior to PBS or CDC delivery. Animals were sacrificed for analyses after 2 , 6 , or 48 hours of IR injury. (B) Representative protein immunoblots of cleaved caspase-3, caspase-3, RIP1, and GAPDH from the normal $(\mathrm{N})$, border $(\mathrm{B})$, and infarct $(\mathrm{I})$ zones of hearts treated with PBS or CDCs. (C) Pooled data from immunoblots, including those in $\mathbf{B}$, reveal a reduction in caspase-3 activation and RIP1 expression levels in the infarct region of CDC-treated hearts ( $n=3-4$ rats per group). (D) Representative images of TUNEL-stained heart tissue from the infarct zones of PBS- and CDC-treated hearts. Scale bar: $50 \mu \mathrm{m}$. (E) Quantitative assessment of cardiomyocytes in D and in similar images reveals reduced TUNEL positivity in CDCtreated hearts at all time points ( $n=3-4$ rats per group). (F) Protein cytokine expression of MMP8 and CXCL7 is elevated in the infarct zone of hearts treated with CDCs ( $n=3-4$ rats per group). Graphs depict mean \pm SEM. Statistical significance was determined using either 1-way or 2-way ANOVA followed by Bonferroni's multiple comparisons test. ${ }^{*} P<0.05$.
CDC-treated hearts showed a reduction in TUNEL ${ }^{+}$cardiomyocytes within the infarct region (Figure 3D), which was most dramatic 2 and 6 hours after IR (Figure 3, D and E). No change in vessel density was observed 48 hours after IR (Supplemental Figure $1, C$ and D). Cytokine protein arrays revealed elevated protein expression of MMP8, which has been associated with wound healing (35) and macrophage inactivation (36), and CXCL7, which is inducibly expressed in monocytes in response to stromal stimulation (ref. 37 and Figure 3F). These were the first hints that macrophages might be involved in the cardioprotective effect of CDCs.

$C D C$ reduce the number of $C D 68^{+}$macrophages within the ischemic heart. To test the idea that CDCs modulate inflammation following IR, we examined the leukocyte profile of peripheral blood and cardiac tissue (Figure 4A). Delivery of CDCs to the heart altered neither circulating leukocytes (Supplemental Table 2) nor serum expression of proinflammatory MCP-1 or IL-4 (Figure $4 \mathrm{E})$. It did, however, reduce specific leukocyte populations within the heart, notably $\mathrm{CD} 45^{+} \mathrm{CD} 68^{+}$macrophages (Figure $4 \mathrm{~B}$ ) and
$\mathrm{CD}_{4} 5^{+} \mathrm{CD} 11 \mathrm{~b}^{+} \mathrm{CD} 11 \mathrm{c}^{+}$dendritic cells (Supplemental Table 3); both are members of the mononuclear phagocyte system. Interestingly, granulocytes $\left(\mathrm{CD} 45^{+} \mathrm{Gran}^{+}\right)$, another acute infiltrating inflammatory cell type (2), were unaltered (Figure 4B). These data were validated using immunohistochemistry to detect CD68 within the infarct region of hearts isolated 2, 6, and 48 hours following IR (Figure 4, C and D). While the $\mathrm{CD}^{+} 8^{+}$cell number was similar in hearts treated with CDC or PBS at 2 and 6 hours after IR, the number of $\mathrm{CD}^{2} 8^{+}$cells (macrophages) was reduced at 48 hours in the hearts of CDC-treated animals (Figure 4, C and D). Furthermore, we did not observe changes in circulating levels of MCP-1 (CCL2) or IL-4 (Figure 4E), which might have accounted for the altered macrophage influx between treatment groups.

Systemic depletion of macrophages using clodronate reduces the efficacy of CDC therapy. Since we observed a decrease in macrophage count within the infarcted myocardium after CDC delivery, we tested whether systemic depletion of macrophages would recapitulate the benefits of CDC therapy. To do so, we adminis- 
A

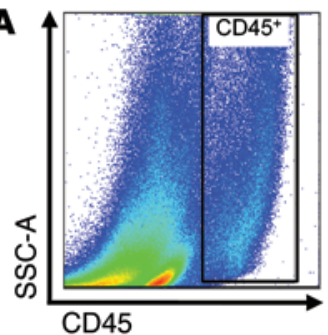

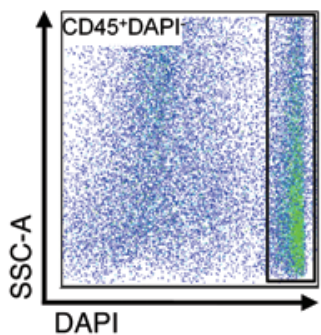

B

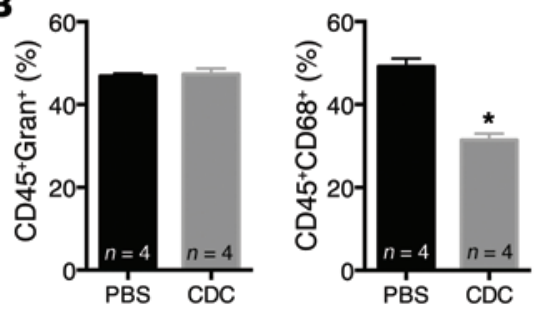

C

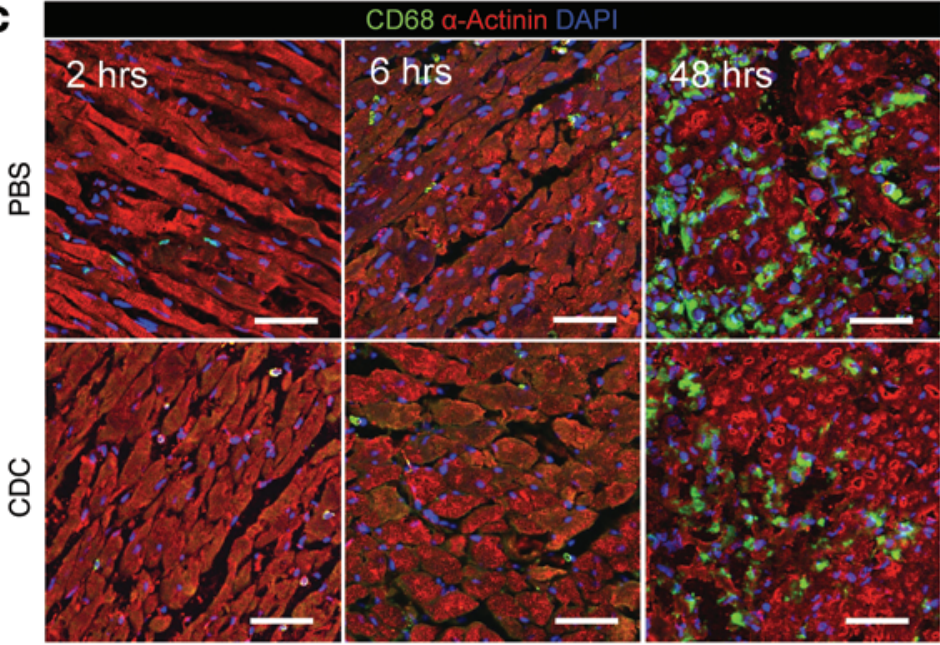

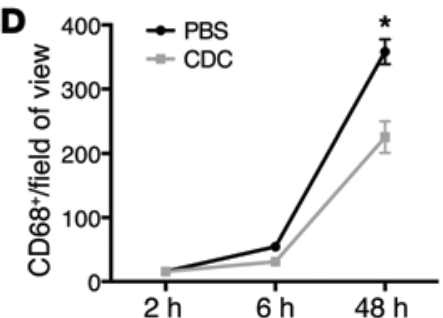

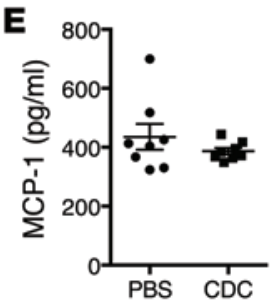

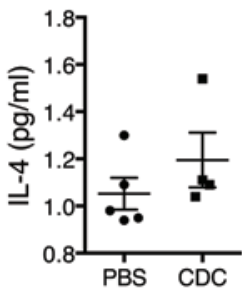

Figure 4. CDC-treated animals have a reduced $\mathrm{CD68}^{+}$macrophage population $\mathbf{4 8}$ hours after IR. (A) Gating strategy for leukocyte identification within the infarcted myocardium prior to subset analysis. CD45+ cells were first identified (FSC-A/CD45'), and then dead cells were excluded (DAPI-). (B) Pooled flow cytometry data from infarcted rat tissue reveal a reduced CD68+ population in CDC- vs. PBS-treated hearts ( $n=4$ rats per group). (C) Immunohistochemical staining of hearts within the infarct zone from CDC- and PBS-treated animals at 2, 6, and 48 hours after IR ( $n=4$ rats per group). Scale bar: $50 \mu \mathrm{m}$. (D) Pooled data from CD68 $8^{+}$cells within the infarct tissue from $C$ at 2,6 , and 48 hours after IR ( $n=4$ rats per group). (E) Concentration of cytokines MCP-1 (CCL2) and IL-4 in sera 48 hours after IR ( $n=4-8$ rats per group). Graphs depict mean \pm SEM. Statistical significance was determined using Student's $t$ test and 2-way ANOVA followed by Bonferroni's multiple comparisons test. ${ }^{*} P<0.05$.

tered clodronate (dichloromethylene diphosphonate [ $\left.\mathrm{Cl}_{2} \mathrm{MDP}\right]$ ) liposomes 24 hours prior to and following IR, using the 20-minute delay protocol from PBS/CDC delivery (Figure 5A). As expected (38), clodronate reduced blood and spleen macrophage populations (Figure 5B). First, we assessed infarct size as an indicator of bioactivity. Clodronate itself did not aggravate IR injury, as we observed no significant difference in infarct mass between the group treated with PBS and the group treated with PBS and $\mathrm{Cl}_{2} \mathrm{MDP}$. However, clodronate attenuated the benefits of CDCs: infarct mass was greater in the group treated with CDCs and $\mathrm{Cl}_{2} \mathrm{MDP}$ relative to that in the CDC-treated group (Figure 5, C and D). The functional changes mirrored those observed pathologically, in that no significant difference in ejection fraction was observed between the group treated with PBS and that treated with CDCs and $\mathrm{Cl}_{2} \mathrm{MDP} 2$ days after IR (Figure 5E). Thus, we rejected the hypothesis that macrophage depletion would recapitulate the benefits of CDC therapy. Instead, the data indicate that macrophages are required for the cardioprotective effects of CDC therapy. Thus, we went on to investigate, in detail, potential effects of CDCs on macrophage phenotype and function. As the $\mathrm{CD} 45^{+} \mathrm{CD} 68^{+}$macrophage population showed the greatest changes in tissue after MI (Figure 4B), we decided to focus on the role of this subpopulation in cardioprotection.
CDCs shift the cardiac $C D 68^{+}$macrophage population away from an M1 phenotype in vivo. It is well recognized that macrophages exhibit the capacity to polarize between M1 and M2 phenotypes $(6,24)$. The M1 population is generally defined by its early infiltration into the myocardium and proinflammatory cytokine expression (e.g., Nos2, Tnf, Il1b, and Il6), while the M2 population is associated with resolution of late-phase inflammation and promotion of tissue repair (e.g., Arg1, Il10, and Pparg). We therefore asked whether CDCs polarize macrophages toward the M1 or M2 phenotype. To do so, we induced MI by permanently ligating the left anterior coronary artery and randomly allocated rats to receive $2 \times 10^{6} \mathrm{CDCs}$ or an equivalent volume of vehicle (PBS) through direct injection at 4 sites in the border zone immediately following infarction. Two days later, hearts were harvested and the infarct and surrounding border tissue were digested. The resulting cell suspension was separated using a density gradient to isolate the mononuclear cell fraction, and then cardiac macrophages were purified by attachment on cell culture dishes (Figure $6 \mathrm{~A}$ ). The $>85 \%$ pure $\mathrm{CD}^{\circ} 8^{+}$populations were then analyzed by qRT-PCR for M1 and M2 gene expression markers (Figure 6B). Interestingly, M1 markers, Nos2, Tnf, and $I l 1 b$, were significantly reduced, but there was no concomitant increase in M2 markers, such as Arg1, Il10, Il4ra, or Pparg. 
A

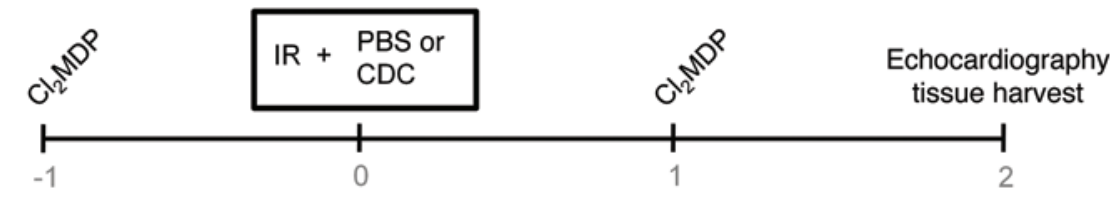

B

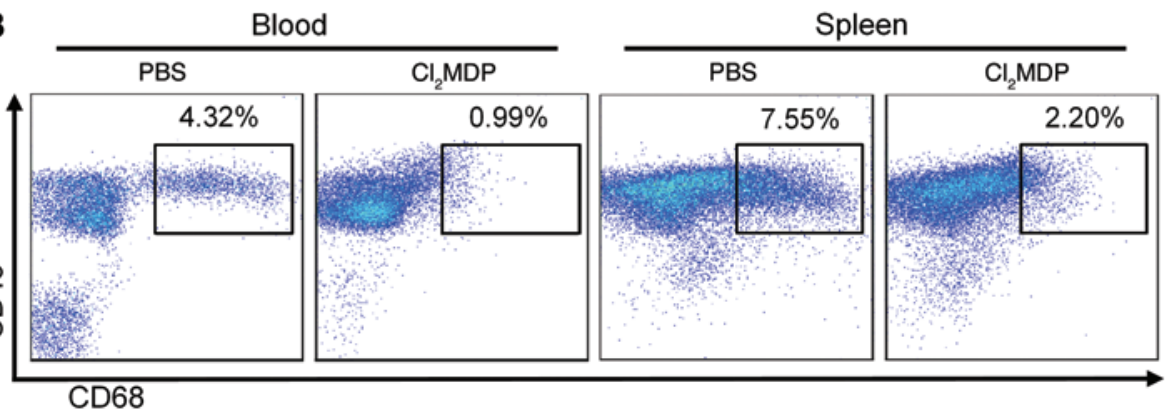

c

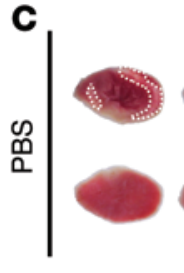

○े
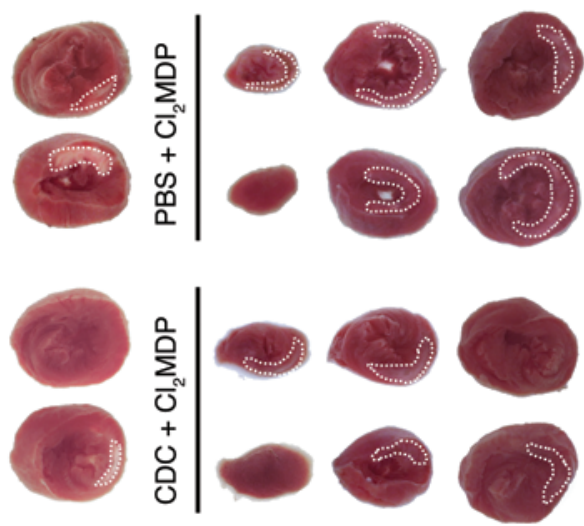

D
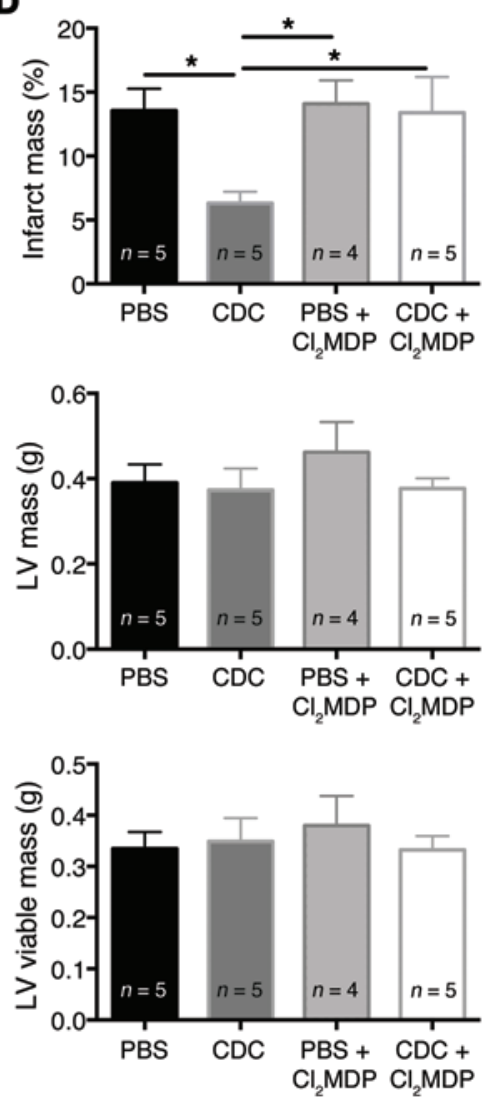

Figure 5. Systemic depletion of endogenous macrophages reduces the efficacy of CDC therapy. (A) Schematic depicting the macrophage depletion protocol using $\mathrm{Cl}_{2} \mathrm{MDP}$ liposomes. Animals were treated with an intravenous infusion of $\mathrm{Cl}_{2} \mathrm{MDP} 1$ day prior to, and one day following, IR and then assessed 48 hours following IR. (B) Representative flow cytometry plots of the $\mathrm{CD} 45^{+} \mathrm{CD} 68^{+}$population in the spleens and blood from $\mathrm{Cl}_{2} \mathrm{MDP}$ - and PBS-treated animals ( $n=5$ rats per group). (C) Representative TTC-stained hearts from $\mathrm{Cl}_{2} \mathrm{MDP}$ - and PBS-treated animals 48 hours after IR. (D) Clodronate treatment led to trends toward an increase in infarct mass and (E) reduction in cardiac ejection fraction in both PBS- and CDC-treated animals relative to their untreated controls ( $n=4-7$ rats per group). Preischemia measurements were acquired prior to $\mathrm{Cl}_{2} \mathrm{MDP}$ treatment. Graphs depict mean $\pm \mathrm{SEM}$. Statistical significance was determined using Student's $t$ test and 1-way ANOVA followed by Bonferroni's multiple comparisons test. ${ }^{*} P<0.05$.

Thus, CDCs reduce the number of $\mathrm{CD} 68^{+}$macrophages in the infarcted myocardium and polarize macrophages away from the M1 phenotype but not toward a classical M2 state.

CDCs polarize thioglycollate-activated macrophages away from an M1 phenotype in vitro. To test whether CDCs have the capac- ity to modulate macrophage polarity indirectly, we devised an in vitro Transwell coculture protocol (Supplemental Figure 2A). With limited macrophage yield from cardiac tissue, we used readily available macrophages derived from the peritoneal cavity following thioglycollate stimulation (39). Although these peritoneal 

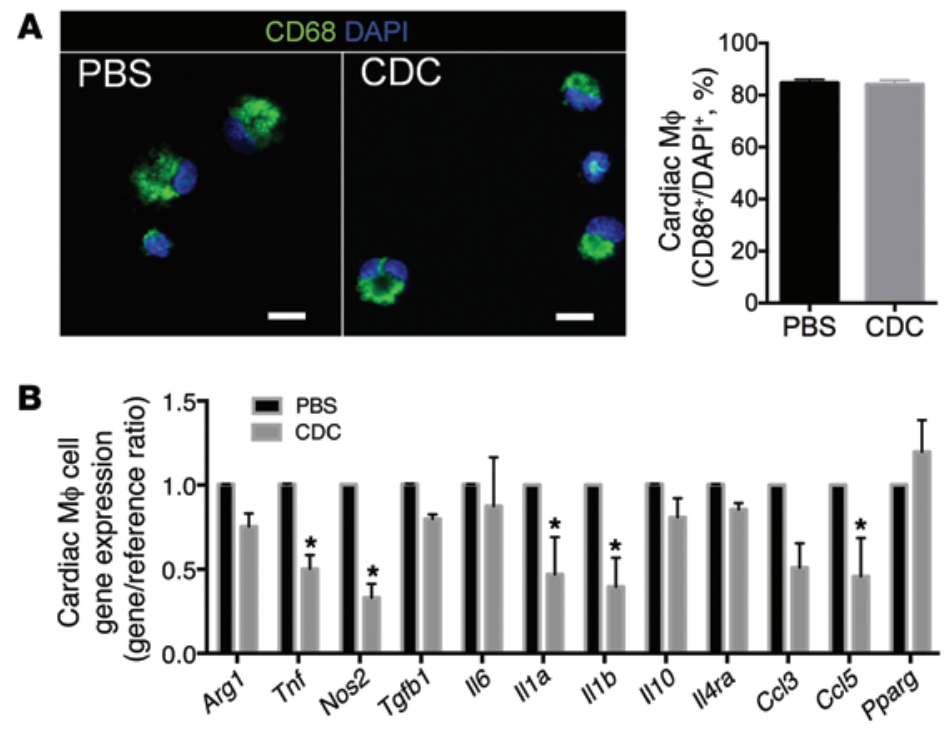

Figure 6. Cardiac macrophages isolated from CDC-treated animals have a distinct cytokine profile. (A) Representative images and pooled quantitative analysis of $\mathrm{CD} 8^{+}$macrophages isolated from cardiac tissue of PBS- and CDC-treated animals 48 hours following MI. Immunohistochemistry reveals a purity level of $>85 \%$ CD68 positivity following cardiac macrophage isolation ( $n=3$ rats per group). Scale bar: $10 \mu \mathrm{m}$. (B) Gene expression profile from cardiac macrophages isolated from infarcted hearts after 48 hours. CDCtreated hearts have cardiac macrophages with reduced M1 (Tnf, Nos2, I/1a, and I/1b), but no change in M2 (Arg1, Tgfb1, I/10, and Pparg), macrophage gene expression. Graphs depict mean \pm SEM Statistical significance was determined using 2-way ANOVA followed by Bonferroni's or Sidak's multiple comparisons test. ${ }^{*} P<0.05$. M $\phi$, macrophage. macrophages are partially activated $(40,41)$, we sought to examine whether CDCs could shift their activation profile away from a proinflammatory state.

A process of peritoneal lavage, red blood cell lysis, and attachment to cell culture plates yielded a highly pure (>90\%) CD68 ${ }^{+}$ mononuclear cell population (Supplemental Figure 2B). Peritoneal macrophages were then preincubated with CDCs in a Transwell coculture system or PBS. After 6 hours of incubation, CDC-primed peritoneal macrophages exhibited reduced M1 gene expression (Il1, Il6, Nos2, and Tnf), without any significant changes in Arg1, Vegfa, or Tgfb1 (Supplemental Figure 2C). Although the gene expression profile was consistent with a reduction in cytotoxic, proinflammatory cytokines, we wanted to test the hypothesis that CDC-primed peritoneal macrophages confer cytoprotection upon distressed ventricular cardiomyocytes without direct cell contact. To do so, we exposed neonatal rat ventricular myocytes (NRVMs) to $50 \mu \mathrm{M} \mathrm{H}_{2} \mathrm{O}_{2}$ and then cocultured them in a Transwell system with CDC- or PBSprimed peritoneal macrophages to simulate an in vivo oxidative stress IR-relevant environment (Supplemental Figure 2A). After 6 hours, we observed reductions in phospho-JNK, phospho-p65, and cleaved caspase-3 activity in NRVMs that had been Transwell cocultured with CDC-primed peritoneal macrophages (Supplemental Figure 2, D and E). Gene expression profiling generally corroborated the protein data. Although not all NRVM genes were concordant, the directional change of a large proportion suggested a protective phenotype, including reduced expression of TLR signaling mediators (Traf6, Irak1, and Irf3) and proinflammatory cytokines (Crp, Il23a, Il6, Nlrp3, and Tnf) (Supplemental Figure 2F).

CDCs polarize unstimulated BM-derived macrophages toward a phenotype distinct from that of either $M 1$ or M2. Following ischemic insult, monocytes are actively recruited from both splenic and $\mathrm{BM}$ reserves and subsequently differentiate into macrophages at the site of injury $(42,43)$. To reproduce the in vivo recruitment of naive/unprimed monocytes to the site of injury in vitro, we isolated $\mathrm{BM}$ cells from femurs, cultured the cells with M-CSF, and then differentiated them into M1 (IFN- $\gamma$ and LPS), M2 (IL-4 and IL-13), $\mathrm{M}_{\mathrm{Fb}}$ (fibroblast Transwell), or $\mathrm{M}_{\mathrm{CDC}}$ (CDC Transwell) macrophages (Figure 7A and Supplemental Figure 3A). To examine whether
$\mathrm{M}_{\mathrm{CDC}}$ macrophages were similar or distinct from $\mathrm{M} 1, \mathrm{M} 2$, or $\mathrm{M}_{\mathrm{Fb}}$ macrophages, we compared the gene expression profiles for known $\mathrm{M} 1$ and M2 genes and general macrophage markers (Figure 7B and Supplemental Figure 3B). As expected, M1 macrophages had elevated Nos2 expression, while M2 macrophages had higher Arg1 and Pparg expression, relative to that of untreated macrophages. $\mathrm{M}_{\mathrm{CDC}}$ macrophages, on the other hand, exhibited reduced Nos 2 and Arg1 relative to both $\mathrm{M} 1$ and $\mathrm{M} 2$ macrophages, indicating that $\mathrm{M}_{\mathrm{CDC}}$ macrophages are polarized to neither a true M1 state nor an M2 state. Of all the genes examined, $\mathrm{M}_{\mathrm{CDC}}$ macrophages expressed the highest level of $I l 1 O$, which encodes an antiinflammatory cytokine.

Two well-established markers for M1 and M2 macrophage polarity are NOS2 and ARG1, respectively (43). The divergent phenotypes involve a common metabolic pathway that converts L-arginine to either L-citrulline and nitric oxide (NOS2 catalysis) or L-ornithine and urea (ARG1 catalysis) (44). Therefore, we examined the relative ratio of NOS2 and ARG1 protein expression. As expected, M2 macrophages exhibited the largest ARG1/NOS2 ratio as well as LYVE-1 and p50 expression, whereas M1 macrophages had the lowest ARG1/NOS2 ratio as well as elevated phospho-p65 expression (Figure 7, $\mathrm{C}$ and D). Interestingly, $\mathrm{M}_{\mathrm{CDC}}$ macrophages have several intermediate protein expression patterns, exhibiting an ARG1/ NOS2 ratio between those of M1 and M2; slightly elevated LYVE-1 relative to untreated, low phospho-p65 (similar to M2); and low p50 expression (similar to M1) (Figure 7, C and D). Flow cytometric analyses of $\mathrm{M}_{\mathrm{CDC}}$ macrophages revealed a reduction in cell size as well as distinct expression of surface markers CD68, CD80, CD86, $\mathrm{CD} 11 \mathrm{~b}$, and CD45 relative to M1, M2, or unstimulated BM-derived macrophages (BMDMs) (Figure 8, A and B). To further characterize and distinguish the functional phenotype of $\mathrm{M}_{\mathrm{CDC}}$ macrophages, we performed a fluorescent microsphere phagocytosis assay (45). Relative to $\mathrm{M} 1$ and $\mathrm{M} 2$ macrophages, $\mathrm{M}_{\mathrm{CDC}}$ macrophages exhibited increased cellular uptake of fluorescent beads following overnight culture (89\% vs. 74\% [M1] or 61\% [M2]) (Figure 8, C and D). Thus, $\mathrm{M}_{\mathrm{CDC}}$ macrophages are functionally distinct from $\mathrm{M} 1$ and $\mathrm{M} 2$ populations, exhibiting higher phagocytic activity.

Together, these data demonstrate that $\mathrm{M}_{\mathrm{CDC}}$ macrophages are distinct from either M1 or M2 macrophages. While clearly differ- 
A
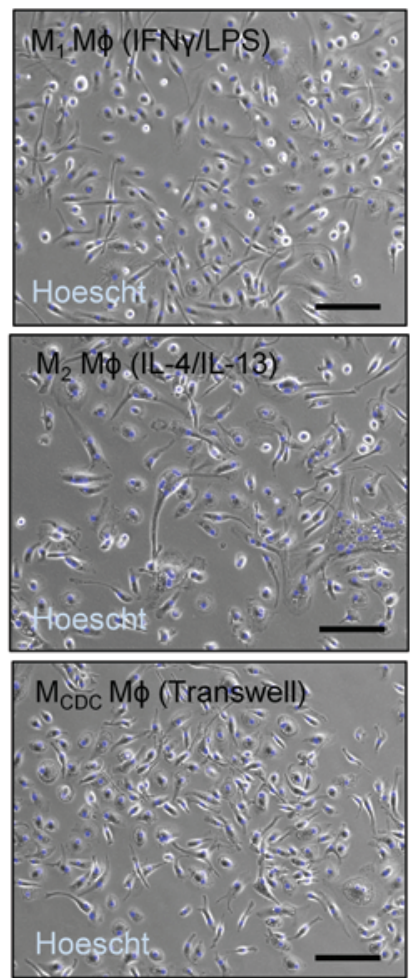

B
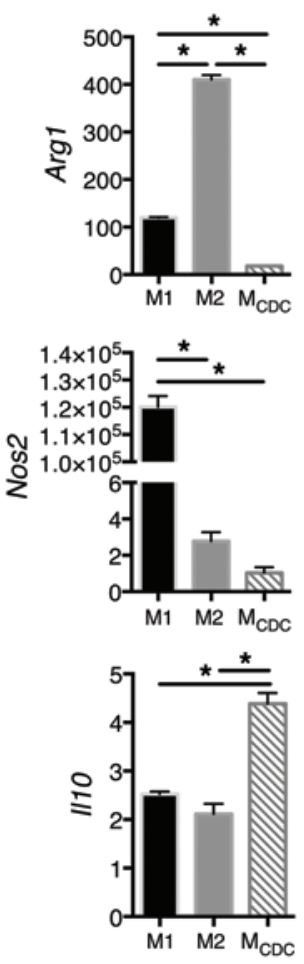
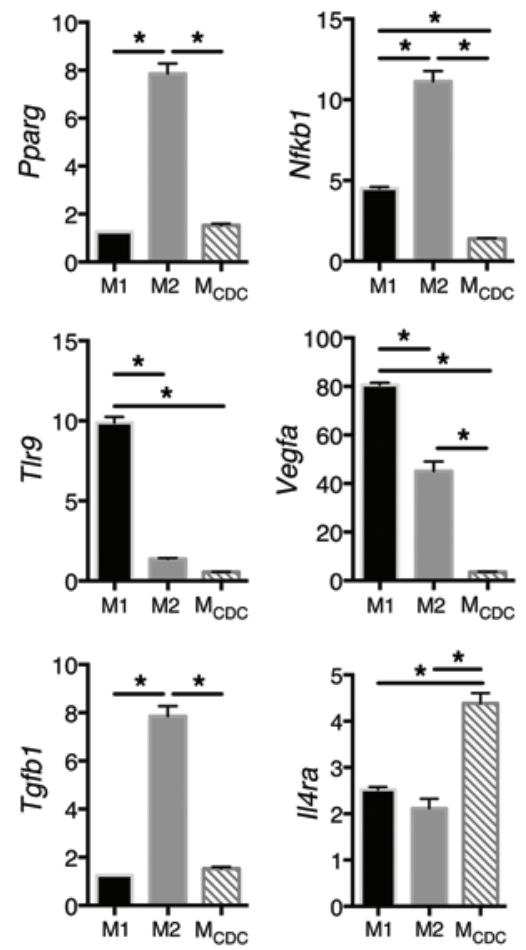

C
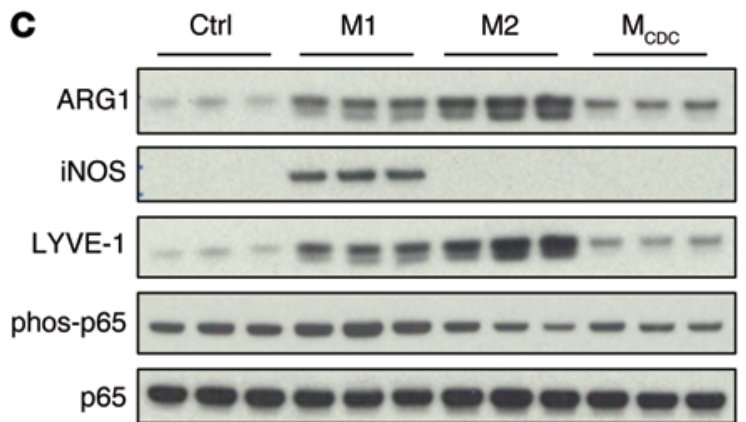

p50

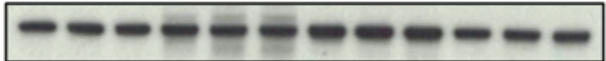

p105

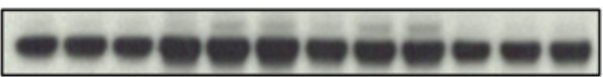

GAPDH

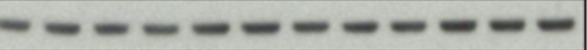

D
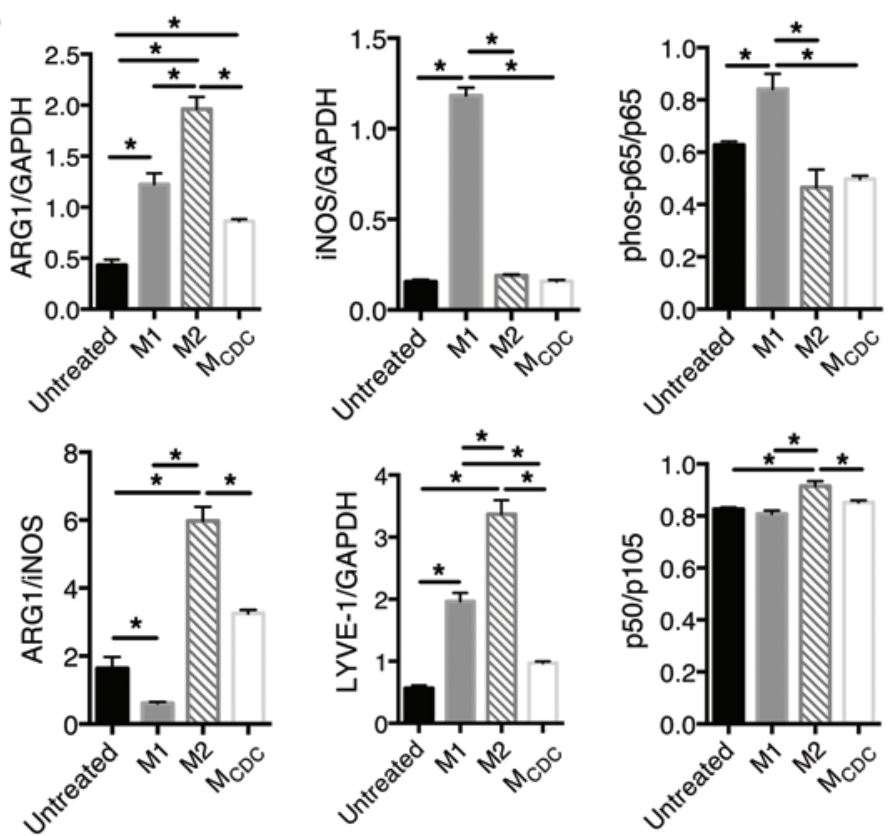

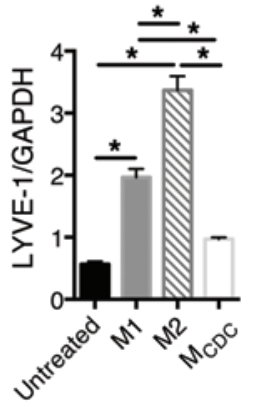

Figure 7. Polarization of BMDMs toward M1, $M 2$, or $\mathrm{M}_{\mathrm{CDC}}$ phenotypes in vitro confers distinct cytokine gene expression and protein marker expression.

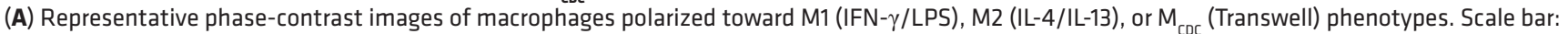
$100 \mu \mathrm{m}$. (B) Gene expression profiles of macrophages polarized toward M1, M2, and $M_{C D C}$ phenotypes ( $n=3$ per group). These data reveal classical upregulation of markers in M1 (Nos2) and M2 (Arg1, Pparg, Nfkb1, and Tgfb1) macrophages, but with a distinct gene expression pattern in $\mathrm{M}_{\mathrm{CDC}}$ (I/10 and II4ra) macrophages. ( $\mathbf{C}$ and $\mathbf{D})$ Protein expression of markers delineating M1 and M2 macrophages vs. $M_{c D C}$ macrophages ( $n=3$ per group). Graphs depict mean \pm SEM. Statistical significance was determined using 1-way ANOVA followed by Tukey's multiple comparisons test. ${ }^{*} P<0.05$.

ent from M1, they are also distinguishable from M2 sub-populations (M2a, M2b, and M2c) (46) based on their low gene expression of Arg1 (in contrast to that of M2a), surface expression of $\mathrm{CD}^{\circ} 6^{+}$(in contrast to that of M2b), and gene expression of $\mathrm{Tg} f b 1$ (in contrast to that of M2c) (Figure 7, B and C, and Figure 8, A and
B). The enhanced phagocytosis further distinguishes $\mathrm{M}_{\mathrm{CDC}}$ macrophages from M1 or M2 macrophages.

$M_{C D C}$ macrophages reduce apoptosis in oxidatively stressed cardiomyocytes in vitro. The recruitment of macrophages to a site of injury results in the phagocytosis of cellular debris and expression 
A
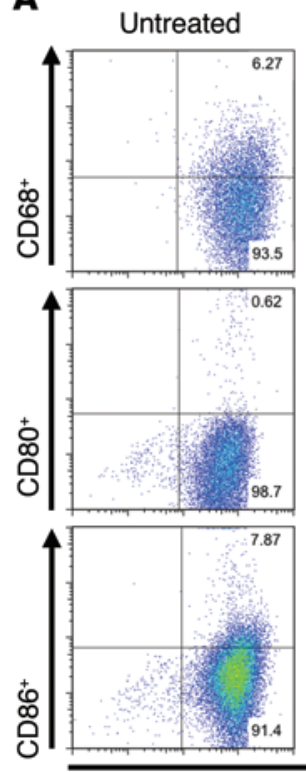

$\mathrm{CD}^{+} 5^{+}$
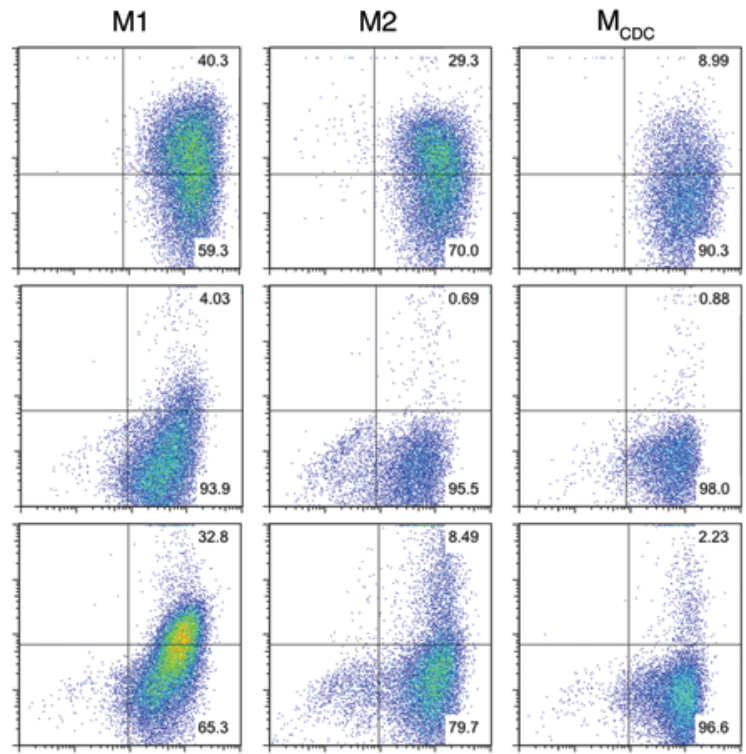

79.7

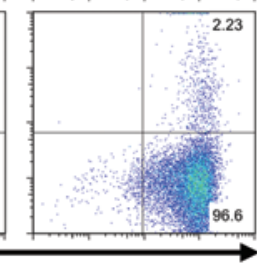

B
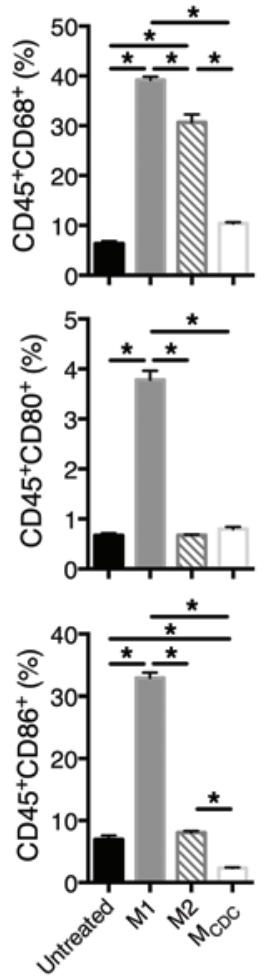
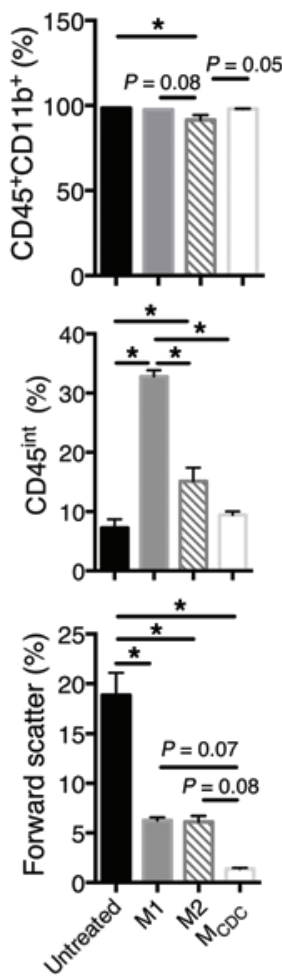

C

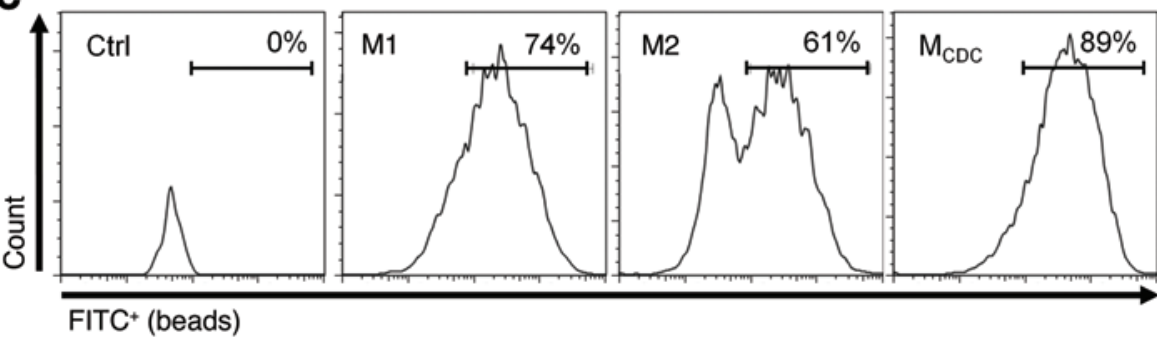

D

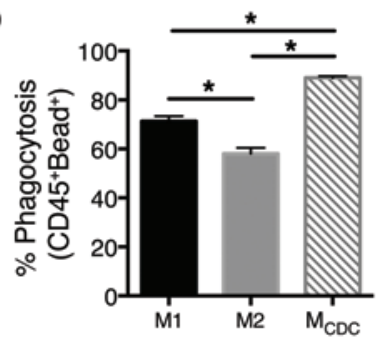

Figure 8. Polarization of BMDMs toward M1, M2, or $M_{C D C}$ phenotypes in vitro confers distinct surface marker expression and phagocytic capacity. (A and B) Surface markers examined by flow cytometry depict reduced CD68, CD80, and CD86 expression and reduced size (forward scatter) on $M_{C D C}$ macrophages ( $n=3$ per group). (C and $\mathbf{D})$ Representative flow cytometry histograms and pooled quantitative analysis of FITC fluorescent bead uptake among macrophage populations ( $n=3$ per group). Graphs depict mean \pm SEM. Statistical significance was determined using 1-way ANOVA followed by Tukey's multiple comparisons test. ${ }^{*} P<0.05$.

of an array of cytokines. As our in vitro gene expression analyses suggested that $\mathrm{M}_{\mathrm{CDC}}$ macrophages secrete a unique cytokine profile, we sought to examine whether $\mathrm{M}_{\mathrm{CDC}}$ macrophages are protective to stressed cardiomyocytes. In in vitro coculture, NRVMs were stressed with $50 \mu \mathrm{M} \mathrm{H}_{2} \mathrm{O}_{2}$ prior to the addition of DiO-labeled M1, $\mathrm{M} 2$, or $\mathrm{M}_{\mathrm{CDC}}$ macrophages (Figure 9A). Cells were examined for viability and number following 6 hours of coincubation. Interestingly, the addition of $\mathrm{M}_{\mathrm{CDC}}$ macrophages to $\mathrm{H}_{2} \mathrm{O}_{2}$-treated NRVMs significantly reduced TUNEL ${ }^{+}$cardiomyocytes and preserved viable cardiomyocytes relative to M1 or M2 macrophages (Figure 9, B-D). At the end of the coculture period we observed differences in the remaining number of macrophages. We found a greater number of $\mathrm{M} 1$ macrophages relative to $\mathrm{M} 2$ or $\mathrm{M}_{\mathrm{CDC}}$ macrophages and increased macrophage TUNEL positivity in M2 macrophages relative to M1 or $M_{C D C}$ macrophages (Figure 9E). Thus, in an oxidative stress model, $\mathrm{M}_{\mathrm{CDC}}$ macrophages do not themselves undergo significant apoptosis but rather limit bystander cardiomyocyte apoptosis. We therefore tested whether $\mathrm{M}_{\mathrm{CDC}}$ macrophages confer cardioprotection in vivo.

Adoptive transfer of $M_{C D C}$ macrophages but not M1 or M2 macrophages simulates $C D C$ therapy after IR. We used adoptive transfer to examine whether $\mathrm{M}_{\mathrm{CDC}}$ macrophages could recapitulate the benefits of CDCs in vivo. To minimize confounding effects of endogenously recruited macrophages, we focused on the time window in which macrophage levels are low in the infarcted myocardium ( $<6$ hours after IR) (Figure 4C). Therefore, we devised a delivery protocol similar to that described earlier in the study but infused vehicle (PBS) or polarized macrophages (M1, M2, or $\mathrm{M}_{\mathrm{CDC}}$ ) rather than CDCs 20 minutes after IR (Figure 10A). All macrophages were labeled with DiI to trace the cells following delivery. After 48 hours of reperfusion, $\mathrm{M}_{\mathrm{CDC}}$ macrophagetreated animals had reduced infarct mass relative to PBS-, M1 macrophage-, and M2 macrophage-treated animals (Figure 10, B 
A

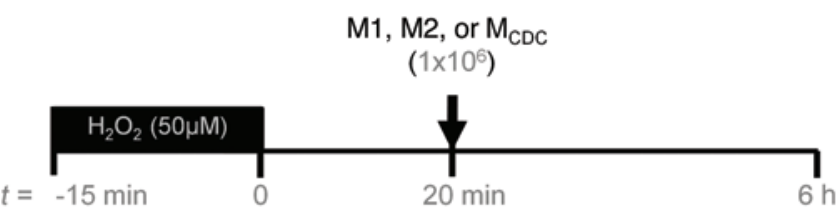

C

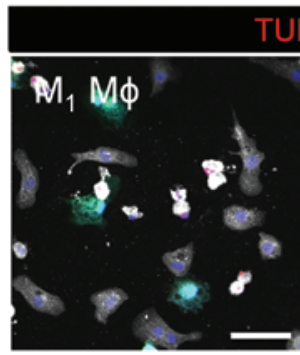

TUNEL M $\phi$-Actinin DAPI
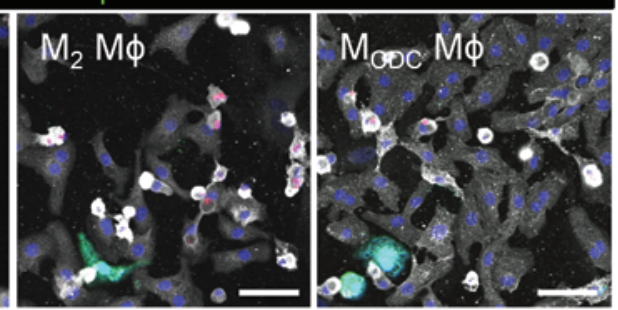

B
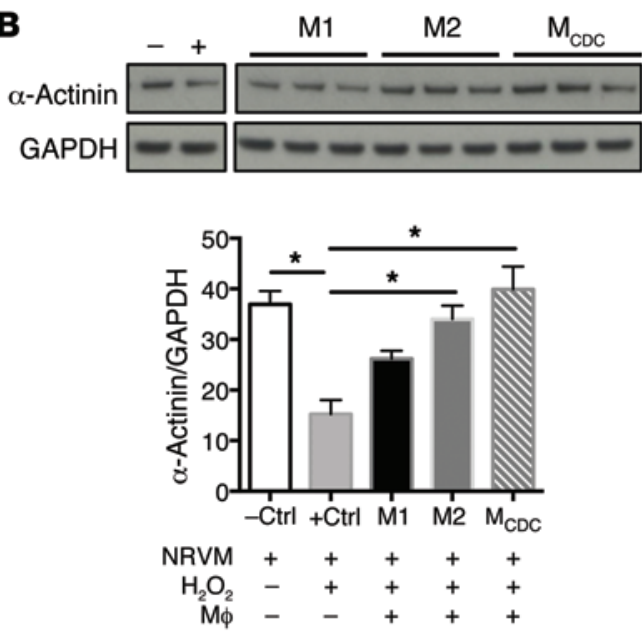

$\mathbf{E}$
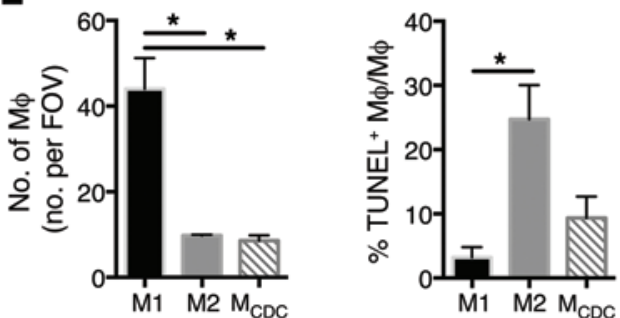

Figure 9. Coculture of $M_{C D C}$ macrophages with oxidatively stressed NRVMs preserves cardiomyocyte viability in vitro. (A) Schematic of in vitro protocol. NRVMs were stressed with $50 \mu \mathrm{M} \mathrm{H}_{2} \mathrm{O}_{2}$ for 15 minutes, serum-free media was replaced for 20 minutes (to simulate reperfusion), and then DiO-labeled M1, $M 2$, or $M_{C D C}$ macrophages were introduced to the NRVMs. After 6 hours, cells were collected for analyses ( $n=3$ per group). (B) Immunoblot of cocultured cells ( $M 1, M 2$, or $\mathrm{M}_{\mathrm{CDC}}$ macrophages with $\mathrm{H}_{2} \mathrm{O}_{2}$-treated NRVMs) and NRVM positive and negative controls (with and without $\mathrm{H}_{2} \mathrm{O}_{2}$, respectively) after 6 hours of culture. The lanes for this blot were run on the same gel but were noncontiguous. (C) Representative images of TUNEL-stained (red) cocultures of M1, M2, or $M_{C D C}$ macrophages (green) with NRVMs (white). Scale bar: $50 \mu \mathrm{m}$. (D) Pooled quantitative analyses of TUNEL+ cardiomyocytes (CM) and viable nucleated cardiomyocytes from M1, M2, and $\mathrm{M}_{\mathrm{CDC}}$ cocultures. (E) Pooled data demonstrating increased macrophage numbers in $\mathrm{M} 1$ cocultures and increased TUNEL ${ }^{+}$macrophages in M2 cocultures. Graphs depict mean \pm SEM. Statistical significance was determined using 1-way ANOVA followed by Tukey's multiple comparisons test. ${ }^{*} P<0.05$.

and C). These macrophages were localized to the border zone and observed in high frequency (Figure 10D).

\section{Discussion}

The gold standard of therapy for acute MI is percutaneous intervention, with the aim of opening the occluded vessel as quickly as possible (47). Nevertheless, reperfusion itself confers some injury to the myocardium $(48,49)$. Several strategies have been used in efforts to reduce the detrimental effects of IR, including ischemic preconditioning (50), but pretreatment is required, limiting realistic utility in patients with MI. A more clinically tractable strategy includes ischemic postconditioning, whereby brief cycles of ischemia imposed during early reperfusion can reduce infarct size (51, 52); but, without immediate manipulation of flow at the time of reperfusion, benefit is lost $(53,54)$. The discovery of cellular postconditioning, whereby CDCs work in MI despite having been administered with some delay after reperfusion, is notable: no other cardioprotective modality successfully reduces infarct size without pretreatment and/or immediate intervention upon reopening the affected artery (55). The idea that cell therapy may mitigate ischemic injury by modulating macrophages is also conceptually novel but should be viewed with caution, given that prior attempts to limit infarct size by manipulating the inflammatory cascade have failed in translation (56). Nevertheless, the benefits seen here are consistent with the immunomodulatory properties recently described for CDCs in other experimental settings $(57,58)$.

Exogenous cell therapy is under development as an adjunctive strategy to treat ischemic heart disease (59-61). The focus, however, has been on subacute or chronic stages of myocardial injury, at which time the opportunity for myocardial preservation has long passed. Here, we have demonstrated that infusion of CDCs into the coronary circulation 20 minutes after IR confers profound cardioprotection to the damaged myocardium (in contrast to the regenerative role of CDCs in established MI; refs. 27, 62-64). This effect is lost, however, if CDC infusion is delayed by 2 hours after IR. These findings are consistent with the observation that reperfusion injury increases over time and in parallel with microvascular obstruction $(47,65)$, which may physically restrict efficient transport of CDCs through the coronary circulation. Inflammation is a critical but poorly understood facet of IR injury (2). With evidence supporting an immunomodulatory role for CDCs in vitro (58) and in a chronic model of MI $(28,31)$, we hypothesized that CDCs would modify the local, innate immune response to confer cardioprotection following IR. 
A

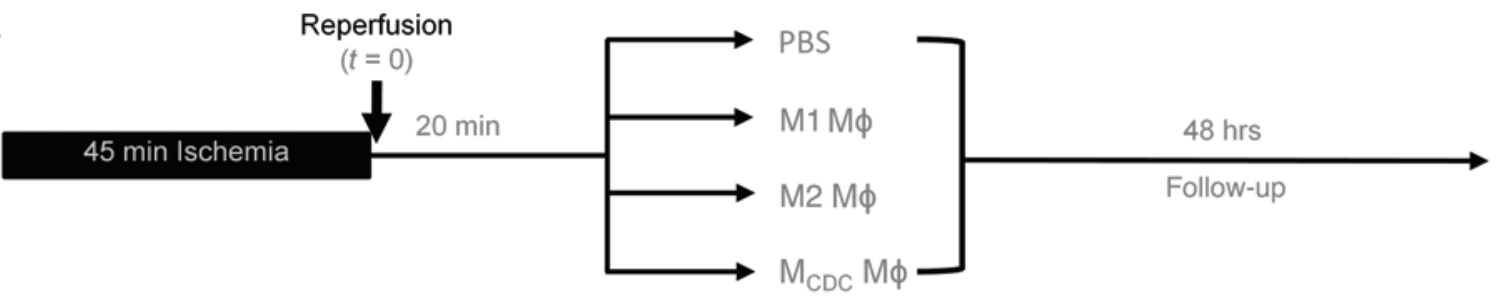

B

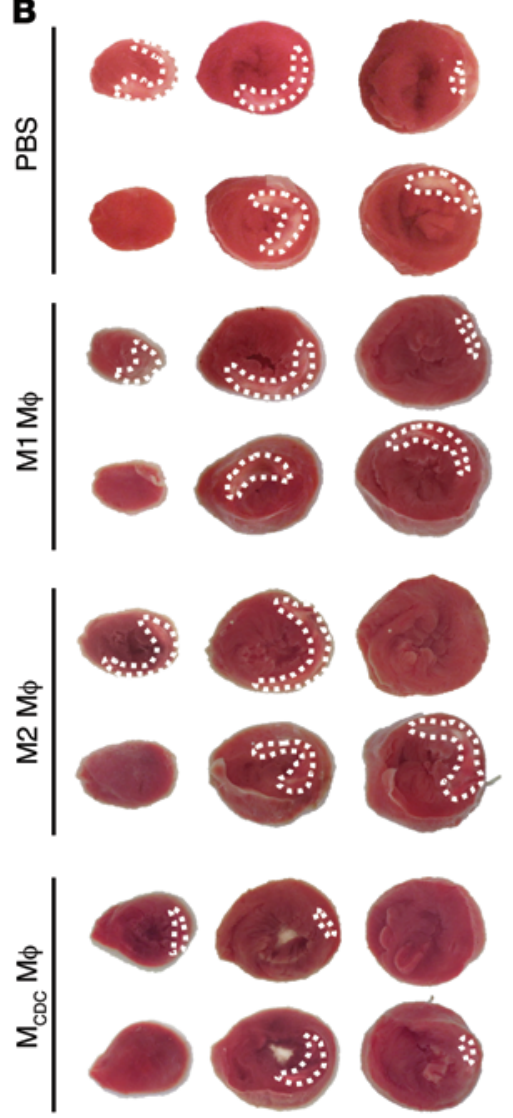

C
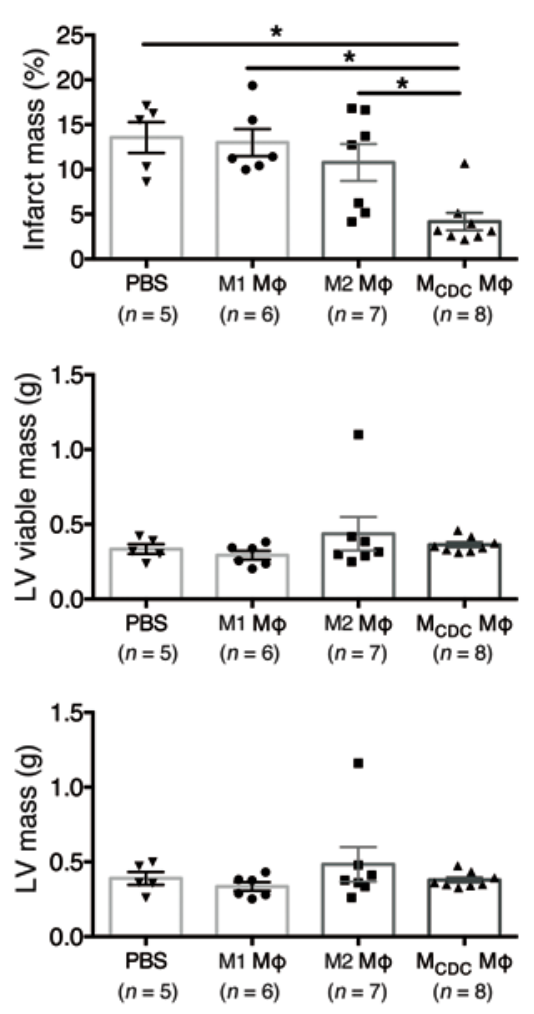

D

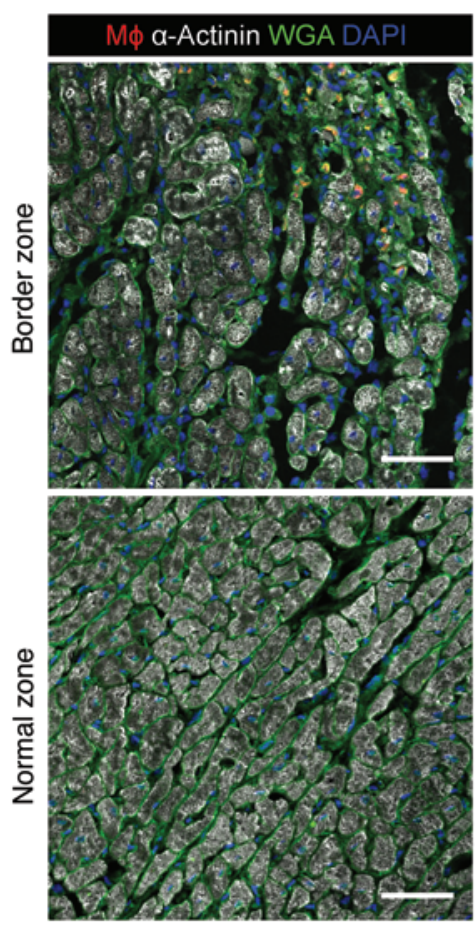

Figure 10. Adoptive transfer of $M_{\mathrm{CDC}}$ macrophages reduces infarct size when administered 20 minutes following reperfusion. (A) Schematic of infusion protocol. Rats underwent 45 minutes of ischemia, followed by 20 minutes of reperfusion prior to administration of PBS or Dil-labeled M1, M2, or $M_{C D C}$ macrophages. Analyses were performed 48 hours after IR injury. (B) Representative images of TTC-stained hearts treated with PBS or M1, M2, or $M_{C D C}$ macrophages. (C) Pooled data demonstrating percentage of infarct mass and LV viable mass, as assessed from TTC-stained hearts ( $n=4-8$ rats per group). (D) Representative image of the localization of Dil-labeled macrophages within the infarct border zone; no Dil-labeled macrophages were observed in the noninfarcted region. Scale bar: $50 \mu \mathrm{m}$. Graphs depict mean \pm SEM. Statistical significance was determined using 1-way ANOVA followed by Tukey's multiple comparisons test. ${ }^{*} P<0.05$.

Macrophages are a populous and highly plastic immune cell source. During the acute phase of inflammation, these cells are either found endogenously within tissues (e.g., skin, brain, liver, and heart) as resident macrophages or recruited from peripheral BM or splenic reserves, as inflammatory macrophages $(6,42)$. Within the heart, at least 4 populations exist at steady state (25). During an inflammatory reaction such as ischemia, Ly $6^{\text {hi }}$ monocytes are rapidly recruited to the site of injury within the heart and support the replenishment of resident cell subpopulations (25). Here, we demonstrate in macrophages from three distinct sources (cardiac, peritoneal, and BM-derived) that CDCs specifically shift macrophages away from a proinflammatory (M1-like) phenotype. Given the low retention rate following coronary infusion $(66,67)$, we propose that CDCs secrete factors that foster a cardioprotective microenvironment with extensive crosstalk between resident and infiltrating cell types and direct antiapoptotic effects on distressed cardiomyocytes $(32,33,62)$. Our data are consistent with the following hypothesis: cellular postconditioning initially works by improving cardiomyocyte viability and modifying resident macrophages, while sustained benefit is mediated by CDC-induced changes in the polarization state of infiltrating macrophages. Exosomes may mediate the CDC effects on macrophages $(62,68)$. We speculate that $\mathrm{M}_{\mathrm{CDC}}$ macrophages are particularly effective in clearing necrotic debris and mitigating subsequent proinflammatory cascades, thereby limiting infarct size $(14,69)$. The observation that phagocytosis is enhanced in CDC-conditioned macro- 
phages is consistent with such a conjecture, but definitive testing will require new lines of experimentation.

Tissue microenvironments have been well described and studied over the past several decades, most notably within the $\mathrm{BM}$ and tumors $(70,71)$. These distinct niches support not only normal stem cell function and therapeutic activation but also malignancy (72). Inflammatory cells, and most specifically macrophages that exist in close proximity to stromal cells and resident stem cells, are essential in maintaining the hematopoietic stem cell niche (73). It is likely that macrophages and stromal cells bidirectionally communicate to support repair in several different tissue microenvironments. For instance, macrophages are necessary for the formation of niches required for limb regeneration in salamanders (74), for skeletal muscle regeneration following toxinmediated injury (75), and for cardiac regeneration in neonatal mice after MI (38). With a growing appreciation for macrophage heterogeneity, it will be important to delineate the factors governing the polarization (denoted by transcriptional regulation and expression of surface markers) of macrophages during ischemic injury $(24,25,43)$. Since resident and inflammatory macrophages derive from distinct progenitor sources (yolk sac vs. BM) (7, 9, 25 ), it is likely that each population is intrinsically distinct but endowed with a capacity to serve functionally redundant roles, as demonstrated through the repopulation of resident cardiac macrophages with Ly6 $\mathrm{C}^{\text {hi }}$ monocytes following ischemic injury (25).

We believe that our results provide novel insight into the mechanism of cellular therapy following ischemic injury. As a result of CDC-induced macrophage priming, macrophages express genes in a pattern that differs from either M1 or M2 (including M2a, M2b, and M2c) states (46). Specifically, we demonstrate not only the capacity to reverse the preactivated, thioglycollate-stimulated peritoneal macrophages away from a cytotoxic phenotype (simulating activated macrophages within the myocardium), but also the directed transition from a more naive state, as observed in our BMDM experiment (simulating recruited macrophages within the myocardium), toward a cytoprotective phenotype. Macrophages primed by CDCs exhibit a distinctive polarization state characterized by the expression of specific genes, cytokines, and membrane markers as well as elevated phagocytic capacity, which together confer cytoprotective properties. $M_{C D C}$ macrophages infused after IR have the endogenous capacity to home to the ischemic border zone, where they reduce infarct size. While our data include endpoints at 2 weeks after IR injury, it would be worthwhile to characterize the longterm cardioprotective effects of CDC therapy to discern whether dysrhythmias and longer-term LV remodeling are affected.

The finding of a macrophage-mediated mechanism of cardioprotection highlights the protean effects of heterogeneous macrophage populations. By favoring one particular polarized macrophage state, CDCs confer therapeutic efficacy when administered after reperfusion, a setting previously believed to be refractory to medical intervention beyond the narrow time window of ischemic postconditioning. The present work defines a promising intervention, targeted at the inflammatory cascade, which can limit myocardial injury. We not only pinpoint macrophages as the key effectors of cellular postconditioning, but also find that macrophages themselves, when appropriately primed, can have therapeutic utility. The fact that functional and structural benefits can be recruited 20 minutes after reperfusion, a time when previous work would suggest that the cascade of death is set in stone $(47,76)$, is noteworthy.

\section{Methods}

\section{Experimental protocols, animals, and surgical procedures}

Seven- to ten-week-old female Wistar-Kyoto (WKY) rats (Charles River Labs) were used for all in vivo experimental protocols. To induce IR injury, rats were provided general anesthesia and then a thoracotomy was performed at the fourth intercostal space to expose the heart and left anterior descending coronary artery (LAD). A 7-0 silk suture was then used to ligate the LAD, which was subsequently removed after 45 minutes to allow for reperfusion. Twenty minutes (or two hours) later, cells (or PBS control) were injected into the LV cavity with an aortic cross-clamp, over a period of 20 seconds. To induce MI, the LAD was permanently ligated and cells (or PBS control) were injected into 4 regions within ischemic border zone.

For our macrophage depletion studies, WKY rats were intravenously injected with $1 \mathrm{ml}(5 \mathrm{mg} / \mathrm{ml}$ ) clodronate liposomes (Clodrosome, Encapsula NanoSciences) 1 day prior to and 1 day following IR injury.

\section{Rat CDC isolation}

Allogeneic CDCs were derived as previously described (26). Briefly, heart tissue from Sprague-Dawley rats (Charles River Labs) was isolated, minced, enzymatically digested, and then plated to allow cardiac explant cell growth. After 7 to 10 days, cells were harvested and plated into a nonadherent cell culture dish to support cardiosphere formation. After 2 days, cardiospheres were isolated and then plated on an adherent dish to allow CDC growth. Cells were subsequently expanded to passage 4 to 6 and used for all experimental work. Based on previously established in vivo dosing studies (67), we used $5 \times 10^{5}$ CDCs resuspended in $100 \mu \mathrm{l}$ PBS (5\% heparin, 1\% nitroglycerin) for treatment after IR and $2 \times 10^{6} \mathrm{CDCs}$ resuspended in $120 \mu \mathrm{l}$ PBS after MI.

\section{Macrophage cell isolation and differentiation}

Cardiac macrophages. WKY rats underwent MI and then were randomly allocated to receive either PBS or CDCs, as described above. After 48 hours, hearts were harvested following perfusion with PBS. The infarct and infarct border zones were isolated, minced, enzymatically digested (Liberase Enzyme, Roche), and then filtered through a 70- $\mu \mathrm{m}$ mesh. Mononuclear cells were isolated using a density gradient (Histopaque 1083, Sigma-Aldrich), washed, resuspended in RPMI (supplemented with $1 \% \mathrm{FBS}$ ), and then plated. Following a 2-hour incubation at $37^{\circ} \mathrm{C}$, $5 \% \mathrm{CO}_{2}$, the attached cardiac macrophage cells were washed with PBS and then incubated with RPMI for downstream analyses.

Peritoneal macrophages. Brewer's Thioglycollate solution (3\% in PBS; Sigma-Aldrich) was injected into the peritoneal cavity of WKY rats. Three days later, macrophage cells were harvested following intraperitoneal lavage with PBS. Cells were filtered through a $70-\mu \mathrm{m}$ mesh, lysed with ACK buffer (Invitrogen), and then resuspended and plated using RPMI. Following a 2-hour incubation at $37^{\circ} \mathrm{C}, 5 \% \mathrm{CO}_{2}$, the attached peritoneal macrophage cells were washed with PBS and then incubated with RPMI for further analyses.

BMDMs. Femurs were isolated from 7- to 10-week-old WKY rats. $\mathrm{BM}$ was isolated, flushed with PBS (containing 1\% FBS, 2 mM EDTA), 
and filtered through a 70- $\mu \mathrm{m}$ mesh. Red blood cells were lysed with ACK buffer (Invitrogen) and resuspended in IMDM (Gibco) containing $10 \mathrm{ng} / \mathrm{ml} \mathrm{M-CSF}$ (eBioscience) for plating. After 3 days, the media were exchanged. BMDMs were incubated on the night between days 7 and 8 ( 18 hours) to polarize toward M1 (100 ng/ml LPS and $50 \mathrm{ng} / \mathrm{ml}$ IFN- $\gamma$; Sigma-Aldrich and R\&D Systems, respectively), M2 (10 ng/ml IL-4 and IL-13; R\&D Systems), $\mathrm{M}_{\mathrm{Fb}}$ (fibroblast Transwell coculture), or $\mathrm{M}_{\mathrm{CDC}}$ (CDC Transwell coculture) macrophages. For in vivo infusion, $1 \times 10^{6}$ BMDMs were labeled with DiI (Vybrant Cell-Labeling Solutions, Invitrogen) according to the manufacturer's protocol and then infused following IR, as described above.

\section{Total leukocyte isolation}

WKY rats underwent IR and then were randomly allocated to receive either PBS or CDC, as described above. After 48 hours, blood was collected from the right atrium in heparinized tubes and hearts were collected following perfusion with PBS.

Peripheral blood. Blood was separated by centrifugation at $1,850 \mathrm{~g}$ for 15 minutes. The buffy coat layer was isolated and resuspended in FACS buffer. Following centrifugation, red blood cells were lysed from the pellet using ACK buffer (Invitrogen). The resulting white blood cells were used for flow cytometric analyses.

Cardiac cell suspension. The infarct and infarct border zones were isolated, minced, digested with Liberase Enzyme (Roche), and then filtered through a $70-\mu \mathrm{m}$ mesh. The resulting cell suspension was used for flow cytometric analyses.

\section{Flow cytometry}

All antibodies used for this study are listed in Supplemental Table 4. Analyses were performed using a CyAn ADP (Beckman Coulter) flow cytometer. Freshly isolated samples were resuspended in FACS buffer (PBS containing 1\% FBS and 2 mM EDTA) and stained with conjugated antibody for 20 to 30 minutes at $4^{\circ} \mathrm{C}$. Cells were washed and resuspended with FACS buffer for flow cytometric analyses in which inflammatory cell populations were designated, following gating/ stratification of their marker profile.

\section{Phagocytosis assay (fluorescent bead microspheres)}

To examine the phagocytic capacity of unstimulated and stimulated macrophage populations, we performed a fluorescent latex bead assay (Cayman Chemical) according to the manufacturer's protocol. Following prestimulation toward M1, M2, or $\mathrm{M}_{\mathrm{CDC}}$ polarities, macrophages were treated overnight ( 18 hours) with FITC fluorescently labeled latex microspheres $(0.1-\mu \mathrm{m}$ diameter). Cells were collected and then stained with CD45-PE-Cy7 (BD Biosciences) and DAPI (Invitrogen) to examine all BM-derived cells and exclude dead cells, respectively. We then performed flow cytometric analysis and immunohistochemistry for quantitation and qualitative assessment of microsphere uptake in each group.

\section{Cardiac functional measurements}

Transthoracic echocardiography (Vevo 770, Visual Sonics) was performed prior to, and following, IR injury at the designated time points (preischemia, 48 hours, 2 weeks). Two-dimensional short and long axes were visualized. Three representative cycles were captured for each animal/time point, and measurements for LV end-systolic dimension, LV end-diastolic dimension, and ejection fraction were obtained and averaged.

\section{Tissue harvest}

Cryostat sectioning. Hearts were arrested in diastole following intraventricular injection of $10 \%$ potassium chloride $(\mathrm{KCl})$ and then excised and washed in PBS. The atria and base above the infarct were removed. The tissue was fixed in $4 \%$ PFA (4\% paraformaldehyde in PBS), processed through a sequential sucrose gradient (10\%, 20\%, 30\% in PBS), embedded in OCT compound (Tissue-Tek OCT), and then kept at $-80^{\circ} \mathrm{C}$ until sectioning. Tissue samples were cut at $5-\mu \mathrm{m}$ thickness.

\section{Histology, immunohistochemistry, and immunocytochemistry}

2,3,5-Triphenyl-2H-tetrazolium chloride. Two days following IR injury, hearts were arrested in diastole following intraventricular injection of $10 \% \mathrm{KCl}$. Hearts were then excised, washed in PBS, and cut into serial sections of about $1 \mathrm{~mm}$ in thickness (from apex to basal edge of infarction). Sections were incubated with 2,3,5-triphenyl-2H-tetrazolium chloride (TTC) (1\% solution in PBS) for 20 minutes in the dark, washed with PBS, and then imaged and weighed. Infarcts were delineated from viable tissue (white vs. red, respectively) and analyzed using ImageJ software. Infarct mass, viable mass, and LV mass were calculated by extrapolating for infarct and noninfarct volumes (based on the areas calculated from both sides of a tissue section) and weight of the tissue. Percentage infarct mass was calculated using the following formula: (infarct mass/viable mass) $\times 100 \%$.

$A A R$. Two days following IR injury, the thoracic cavity was reopened and the LAD was religated at the same site of occlusion. Rats were then injected intravenously with gentian violet stain to perfuse the myocardium and delineate the nonreperfused myocardium. Hearts were arrested in diastole $(10 \% \mathrm{KCl})$, excised, washed in PBS, and cut into serial 1-mm-thick sections (from apex to basal edge of infarction) for TTC staining. The AAR was determined by delineating the blue (perfused) from the red (nonperfused) myocardium and analyzed by Image J software. Percentage AAR was calculated using the following formula: $(\mathrm{AAR} / \mathrm{LV}$ area $) \times 100 \%$; percentage infarct was calculated using the following formula: (infarct area/AAR) $\times 100 \%$.

Masson's trichrome and Picrosirius red staining. OCT-cut tissue was stained according to the manufacturer's protocol (Sigma-Aldrich) and then mounted and imaged. Morphometric analyses of the infarcted tissue were performed using ImageJ software. Infarct thickness and size measurements were obtained from the midpapillary level of the infarcted heart. Infarct fibrosis measurements were obtained at the base, midpapillary, and apical regions of the infarct heart.

Immunohistochemistry. For analyses of cardiomyocyte size and inflammatory cell distribution, OCT-embedded tissue sections were fixed with $4 \%$ PFA and stained with the following primary antibodies for confocal microscopy: mouse anti-rat $\alpha$-actinin (SigmaAldrich), mouse anti-rat CD68 (AbD Serotec), and mouse anti-rat CD45 (BD Pharmingen). The appropriate fluorescently conjugated secondary antibodies (Invitrogen) were applied prior to mounting using Fluoroshield with DAPI (Sigma-Aldrich). To detect apoptotic cardiomyocytes, we performed a TdT dUDP Nick-End Labeling Assay (TUNEL, Roche) according to the manufacturer's protocol and stained with $\alpha$-actinin and DAPI. To determine cardiomyocyte size, we used an Alexa Fluor 488-conjugated wheat-germ agglutinin (Invitrogen Life Technologies) stain in conjunction with $\alpha$-actinin and DAPI. Only cardiomyocytes with centrally located nuclei were used for cell size determination. 
Immunocytochemistry. Peritoneal and cardiac macrophage cells were cultured on fibronectin-coated slides, fixed with $4 \%$ PFA, and stained with mouse anti-rat CD68 (AbD Serotec). The appropriate fluorescently conjugated antibody was added, and then cells were counterstained with DAPI. For live cultured BMDM cells, Hoechst 33342 (Sigma-Aldrich 14533) was used to distinguish nucleated/multinucleated cells.

\section{Protein and RNA isolation}

Protein. At the appropriate time point following surgery, the heart was harvested and rinsed in PBS. The border, infarct, and normal zones were dissected; placed in Allprotect Tissue Reagent (QIAGEN); and stored at $-80^{\circ} \mathrm{C}$ until use. Tissues were minced, suspended in T-PER (with HALT protease and phosphatase inhibitors, Thermo Scientific), and homogenized with a bead ruptor. For cell culture experiments, cells were lysed with RIPA (with HALT protease and phosphatase inhibitors, Thermo Scientific), scraped off culture plates, and sonicated for 3 cycles of 10-second bursts (Active Motif) on ice. The resulting suspensions were centrifuged at $10,000 \mathrm{~g}$ for 15 minutes at $4^{\circ} \mathrm{C}$, and the protein supernatant was collected. Protein concentrations were measured using a BCA assay (Thermo Scientific).

$R N A$. At the appropriate time points, cells were washed and collected for RNA isolation using an RNeasy Mini Kit (QIAGEN) according to the manufacturer's protocol. RNA concentration and purity were determined using a NanoDrop Spectrophotometer (Thermo Scientific).

\section{Quantitative RT-PCR}

To compare the gene expression levels among cells at rest, following coculture or after stimulation, we used both SYBR Green and TaqMan technologies (Applied Biosystems).

SYBR Green. To assess gene expression, cDNA was synthesized from mRNA using a RT ${ }^{2}$ First Strand Synthesis Kit (QIAGEN) according to the manufacturer's protocol. The resulting CDNA was standardized across samples and loaded into the predesigned $\mathrm{RT}^{2}$ Profiler PCR Array (QIAGEN) plates. Gene expression was then amplified over the course of 40 cycles and analyzed by ddCt.

TaqMan. To assess gene expression, cDNA was synthesized from mRNA using the High-Capacity cDNA Reverse Transcription Kit (Applied Biosystems) according to the manufacturer's protocol. The resulting cDNA was standardized across samples and then mixed with master mix and designated primer sets (Life Technologies, Invitrogen). Predesigned TaqMan primer sets for the following genes were purchased from Life Technologies: Arg1, Tnf, Nos2, Tgfb1, Il1a, Il1b, Il6, Il1O, Il4ra, Ccl3, Ccl5, Pparg, Nfkb1, Vegfa, Nod2, and Tlr9.

\section{Western blot analysis and protein array}

Protein samples were prepared for gel electrophoresis (NuPAGE $4 \%-12 \%$ Bis-Tris, Invitrogen) according to the manufacturer's protocol. For all experiments, a normalized final loading concentration of between 10 and $30 \mu \mathrm{g}$ per well was used prior to separation. Proteins were then transferred to a polyvinylidene fluoride membrane (BioRad) for immunoblotting with designated antibodies. Bands were visualized following activation with ECL (Thermo Scientific) and exposure on film (Kodak Carestream Biomax, Sigma-Aldrich).

Rat cytokines were analyzed on a protein array (Raybiotech) according to the manufacturer's protocol. Briefly, tissue lysates were incubated with the antibody array, membranes washed, and then a secondary biotinylated antibody was introduced. Incubation with streptavidin and subsequent exposure with a detection buffer allowed for visualization of dots on film (Kodak Carestream Biomax, Sigma-Aldrich).

\section{Cytokine bead array for serum cytokine analysis}

Serum levels of cytokines were analyzed with a FlowCytomix Multiplex bead array (eBioscience) according to the manufacturer's protocol. Briefly, blood was collected from rats after 48 hours after IR. Sera were then separated by centrifugation and incubated with antibodycoated beads (MCP-1, IFN- $\gamma$, IL-1a, IL-4, TNF- $\alpha$ ). After the appropriate labeling, beads were resuspended with buffer then analyzed using a CyAn ADP (Beckman Coulter) flow cytometer.

\section{NRVM isolation and in vitro assay}

NRVMs were cultured as previously described (77). Briefly, hearts were harvested from 2-day-old Sprague-Dawley rats. Ventricles were isolated, minced, and then enzymatically digested in a solution of trypsin and collagenase overnight. Cells were then resuspended in m199 media (10\% FBS, glucose, penicillin, vitamin $B_{12}$, HEPES, and MEM nonessential amino acids [Gibco]) and preplated to allow noncardiomyocyte cells to attach. The resulting NRVM suspension was collected and counted prior to plating for experimental use.

To induce oxidative stress in NRVMs, $10 \mathrm{M} \mathrm{H}_{2} \mathrm{O}_{2}$ (Sigma-Aldrich) was diluted in IMDM (Gibco) to a final concentration of $50 \mu \mathrm{M}$. Cells were then incubated for 15 minutes at $37^{\circ} \mathrm{C}$ prior to media exchange. For in vitro NRVM-macrophage coculture, macrophages were dyed with DiO (Vybrant Cell-Labeling Solutions, Invitrogen) for 3 minutes at $37^{\circ} \mathrm{C}$, washed with FACS buffer, resuspended in IMDM, and then added to the NRVM culture dish.

\section{Statistics}

Results are expressed as mean \pm SEM. To determine differences between groups at a single time point, data were tested using either 2-tailed, unpaired, Student's $t$ test or 1-way ANOVA followed by Tukey's multiple comparisons test. To determine differences between groups at multiple time points, data were tested using 2-way ANOVA followed by Sidak's or Bonferroni's multiple comparisons test. All analyses were performed using Prism 5 software (GraphPad), and only differences with a $P$ value of less than 0.05 were considered statistically significant.

\section{Study approval}

The Institutional Animal Care and Use Committee of Cedars-Sinai Medical Center (Los Angeles, California, USA) approved all animal care and experimental procedures prior to commencement of this study.

\section{Acknowledgments}

We would like to thank Patricia Lin and Carolina Castillo for assistance with flow cytometry and tissue sectioning, Hee Cheol Cho and Eugenio Cingolani for helpful discussions, P.K. Shah for comments on the manuscript, and Elizabeth Kim, Eli Simsolo, and Ryan Middleton for technical assistance. This work was supported by the Board of Governors of the Cedars-Sinai Medical Center. General laboratory support was provided by grants to E. Marbán from the NIH and the Department of Defense.

Address correspondence to: Eduardo Marbán, 8700 Beverly Blvd., Los Angeles, California 90048, USA. Phone: 310.423.7557; E-mail: eduardo.marban@cshs.org. 
1. Frangogiannis NG. Regulation of the inflammatory response in cardiac repair. Circ Res. 2012;110(1):159-173.

2. Frangogiannis NG. The inflammatory response in myocardial injury, repair, and remodelling. Nat Rev Cardiol. 2014;11(5):255-265.

3. Entman ML, et al. Inflammation in the course of early myocardial ischemia. FASEB $J$. 1991;5(11):2529-2537.

4. Jordan JE, Zhao ZQ, Vinten-Johansen J. The role of neutrophils in myocardial ischemia-reperfusion injury. Cardiovasc Res. 1999;43(4):860-878.

5. Simpson PJ, Lucchesi BR. Free radicals and myocardial ischemia and reperfusion injury. J Lab Clin Med.1987;110(1):13-30.

6. Murray PJ, Wynn TA. Protective and pathogenic functions of macrophage subsets. Nat Rev Immunol. 2011;11(11):723-737.

7. Yona S, et al. Fate mapping reveals origins and dynamics of monocytes and tissue macrophages under homeostasis. Immunity. 2013;38(1):79-91.

8. Davies LC, et al. Distinct bone marrow-derived and tissue-resident macrophage lineages proliferate at key stages during inflammation. Nat Commun. 2013;4:1886.

9. Hashimoto D, et al. Tissue-resident macrophages self-maintain locally throughout adult life with minimal contribution from circulating monocytes. Immunity. 2013;38(4):792-804.

10. Jenkins SJ, et al. Local macrophage proliferation, rather than recruitment from the blood, is a signature of TH2 inflammation. Science. 2011;332(6035):1284-1288.

11. Davies LC, Rosas M, Smith PJ, Fraser DJ, Jones SA, Taylor PR. A quantifiable proliferative burst of tissue macrophages restores homeostatic macrophage populations after acute inflammation. Eur J Immunol. 2011;41(8):2155-2164.

12. Geissmann F, Jung S, Littman DR. Blood monocytes consist of two principal subsets with distinct migratory properties. Immunity. 2003;19(1):71-82.

13. Hume DA, Ross IL, Himes SR, Sasmono RT, Wells CA, Ravasi T. The mononuclear phagocyte system revisited. J Leukoc Biol. 2002;72(4):621-627.

14. Nahrendorf $M$, et al. The healing myocardium sequentially mobilizes two monocyte subsets with divergent and complementary functions. JExp Med. 2007;204(12):3037-3047.

15. Saxena A, et al. IL-1 induces proinflammatory leukocyte infiltration and regulates fibroblast phenotype in the infarcted myocardium. J Immunol. 2013;191(9):4838-4848.

16. Swirski FK, et al. Identification of splenic reservoir monocytes and their deployment to inflammatory sites. Science. 2009;325(5940):612-616.

17. Lee WW, et al. PET/MRI of inflammation in myocardial infarction. J Am Coll Cardiol. 2012;59(2):153-163.

18. Troidl C, et al. Classically and alternatively activated macrophages contribute to tissue remodelling after myocardial infarction. J Cell Mol Med. 2009;13(9B):3485-3496.

19. Frantz S, et al. Monocytes/macrophages prevent healing defects and left ventricular thrombus formation after myocardial infarction. FASEB $J$. 2013;27(3):871-881.

20. van Amerongen MJ, Harmsen MC, van Rooijen
N, Petersen AH, van Luyn MJ. Macrophage depletion impairs wound healing and increases left ventricular remodeling after myocardial injury in mice. Am J Pathol. 2007;170(3):818-829.

21. Mills CD, Kincaid K, Alt JM, Heilman MJ, Hill AM. M-1/M-2 macrophages and the Th1/Th2 paradigm. JImmunol. 2000;164(12):6166-6173.

22. Miron VE, et al. M2 microglia and macrophages drive oligodendrocyte differentiation during CNS remyelination. Nat Neurosci. 2013;16(9):1211-1218.

23. Martinez FO, Gordon S, Locati M, Mantovani A. Transcriptional profiling of the human monocyte-to-macrophage differentiation and polarization: new molecules and patterns of gene expression. J Immunol. 2006;177(10):7303-7311.

24. Mosser DM, Edwards JP. Exploring the full spectrum of macrophage activation. Nat Rev Immunol. 2008;8(12):958-969.

25. Epelman S, et al. Embryonic and adult-derived resident cardiac macrophages are maintained through distinct mechanisms at steady state and during inflammation. Immunity. 2014;40(1):91-104.

26. Smith RR, et al. Regenerative potential of cardiosphere-derived cells expanded from percutaneous endomyocardial biopsy specimens. Circulation. 2007;115(7):896-908.

27. Makkar RR, et al. Intracoronary cardiosphere-derived cells for heart regeneration after myocardial infarction (CADUCEUS): a prospective, randomised phase 1 trial. Lancet. 2012;379(9819):895-904.

28. Malliaras K, et al. Safety and efficacy of allogeneic cell therapy in infarcted rats transplanted with mismatched cardiosphere-derived cells. Circulation. 2012;125(1):100-112.

29. Malliaras K, et al. Cardiomyocyte proliferation and progenitor cell recruitment underlie therapeutic regeneration after myocardial infarction in the adult mouse heart. EMBO Mol Med. 2013;5(2):191-209.

30. Tseliou E, et al. Angiogenesis, cardiomyocyte proliferation and anti-fibrotic effects underlie structural preservation post-infarction by intramyocardially-injected cardiospheres. PLoS One. 2014;9(2):e88590.

31. Tseliou E, et al. Allogeneic cardiospheres safely boost cardiac function and attenuate adverse remodeling after myocardial infarction in immunologically mismatched rat strains. J Am Coll Cardiol. 2013;61(10):1108-1119.

32. Kanazawa $\mathrm{H}$, et al. Cellular postconditioning allogeneic cardiosphere-derived cells reduce infarct size and attenuate microvascular obstruction when administered after reperfusion in pigs with acute myocardial infarction. Circ Heart Fail. 2015;8(2):322-332.

33. Chimenti I, et al. Relative roles of direct regeneration versus paracrine effects of human cardiosphere-derived cells transplanted into infarcted mice. Circ Res. 2010;106(5):971-980.

34. Malliaras K, et al. Stimulation of endogenous cardioblasts by exogenous cell therapy after myocardial infarction. $E M B O \mathrm{Mol} \mathrm{Med}$. 2014;6(6):760-777.

35. Gutierrez-Fernandez A, et al. Increased inflammation delays wound healing in mice deficient in collagenase-2 (MMP-8). FASEB J. 2007;21(10):2580-2591.

36. Quintero PA, Knolle MD, Cala LF, Zhuang Y, Owen CA. Matrix metalloproteinase-8 inactivates macrophage inflammatory protein- 1 alpha to reduce acute lung inflammation and injury in mice. J Immunol. 2010;184(3):1575-1588.

37. Pillai MM, Iwata M, Awaya N, Graf L, TorokStorb B. Monocyte-derived CXCL7 peptides in the marrow microenvironment. Blood. 2006;107(9):3520-3526.

38. Aurora AB, et al. Macrophages are required for neonatal heart regeneration. JClin Invest. 2014;124(3):1382-1392.

39. Lagasse E, Weissman IL. Flow cytometric identification of murine neutrophils and monocytes. JImmunol Methods. 1996;197(1-2):139-150.

40. Michl J, Pieczonka MM, Unkeless JC, Silverstein SC. Effects of immobilized immune complexes on Fc- and complement-receptor function in resident and thioglycollate-elicited mouse peritoneal macrophages. J Exp Med. 1979;150(3):607-621.

41. Norton JM, Munck A. Glucose transport in murine macrophages: in vitro characterization of the monosaccharide transport system of the thioglycollate-elicited mouse peritoneal macrophage. J Immunol. 1980;125(1):252-258.

42. Shi C, Pamer EG. Monocyte recruitment during infection and inflammation. Nat Rev Immunol. 2011;11(11):762-774.

43. Lawrence T, Natoli G. Transcriptional regulation of macrophage polarization: enabling diversity with identity. Nat Rev Immunol. 2011;11(11):750-761.

44. Bronte V, Zanovello P. Regulation of immune responses by L-arginine metabolism. Nat Rev Immunol. 2005;5(8):641-654.

45. Dunn PA, Eaton WR, Lopatin ED, McEntire JE, Papermaster BW. Lymphokine-stimulated macrophage phagocytosis of fluorescent microspheres: a rapid new assay. JImmunol Methods. 1983;64(1-2):71-83.

46. Mantovani A, Sica A, Sozzani S, Allavena P, Vecchi A, Locati $M$. The chemokine system in diverse forms of macrophage activation and polarization. Trends Immunol. 2004;25(12):677-686.

47. Yellon DM, Hausenloy DJ. Myocardial reperfusion injury. N EnglJ Med. 2007;357(11):1121-1135

48. Braunwald E, Kloner RA. Myocardial reperfusion: a double-edged sword? J Clin Invest. 1985;76(5):1713-1719.

49. Gottlieb RA, Burleson KO, Kloner RA, Babio BM, Engler RL. Reperfusion injury induces apoptosis in rabbit cardiomyocytes. JClin Invest. 1994;94(4):1621-1628.

50. Murry CE, Jennings RB, Reimer KA. Preconditioning with ischemia: a delay of lethal cel injury in ischemic myocardium. Circulation. 1986;74(5):1124-1136

51. Staat $\mathrm{P}$, et al. Postconditioning the human heart. Circulation. 2005;112(14):2143-2148.

52. Zhao ZQ, et al. Inhibition of myocardial injury by ischemic postconditioning during reperfusion: comparison with ischemic preconditioning. Am J Physiol Heart Circ Physiol. 2003;285(2):H579-H588.

53. Kin $\mathrm{H}$, et al. Postconditioning attenuates myocardial ischemia-reperfusion injury by inhibiting events in the early minutes of reperfusion. Car- 
diovasc Res. 2004;62(1):74-85.

54. Yang XM, Proctor JB, Cui L, Krieg T, Downey JM, Cohen MV. Multiple, brief coronary occlusions during early reperfusion protect rabbit hearts by targeting cell signaling pathways. J Am Coll Cardiol. 2004;44(5):1103-1110.

55. Kloner RA. Current state of clinical translation of cardioprotective agents for acute myocardial infarction. Circ Res. 2013;113(4):451-463.

56. Ray WA, Stein CM, Hall K, Daugherty JR, Griffin MR. Non-steroidal anti-inflammatory drugs and risk of serious coronary heart disease: an observational cohort study. Lancet. 2002;359(9301):118-123.

57. Aminzadeh MA, Tseliou E, Sun B, Cheng K, Malliaras K, Makkar RR, Marban E. Therapeutic efficacy of cardiosphere-derived cells in a transgenic mouse model of non-ischaemic dilated cardiomyopathy. Eur Heart J. 2014;36(12):751-762.

58. Lauden L, et al. Allogenicity of human cardiac stem/progenitor cells orchestrated by programmed death ligand 1. Circ Res. 2013;112(3):451-464.

59. Garbern JC, Lee RT. Cardiac stem cell therapy and the promise of heart regeneration. Cell Stem Cell. 2013;12(6):689-698.

60. Ye L, et al. Cardiac repair in a porcine model of acute myocardial infarction with human induced pluripotent stem cell-derived cardiovascular cells. Cell Stem Cell. 2014;15(6):750-761.

61. Ye L, Zimmermann WH, Garry DJ, Zhang J. Patching the heart: cardiac repair from within and outside. Circ Res. 2013;113(7):922-932.

62. Ibrahim AG, Cheng K, Marbán E. Exosomes as critical agents of cardiac regeneration triggered by cell therapy. Stem Cell Reports. 2014;2(5):606-619.

63. Malliaras K, et al. Validation of contrast-

enhanced magnetic resonance imaging to monitor regenerative efficacy after cell therapy in a porcine model of convalescent myocardial infarction. Circulation. 2013;128(25):2764-2775.

64. Johnston PV, et al. Engraftment, differentiation, and functional benefits of autologous cardiosphere-derived cells in porcine ischemic cardiomyopathy. Circulation. 2009;120(12):1075-1083.

65. Rochitte CE, et al. Magnitude and time course of microvascular obstruction and tissue injury after acute myocardial infarction. Circulation. 1998;98(10):1006-1014.

66. Cheng K, Li TS, Malliaras K, Davis DR, Zhang Y, Marban E. Magnetic targeting enhances engraftment and functional benefit of iron-labeled cardiosphere-derived cells in myocardial infarction. Circ Res. 2010;106(10):1570-1581.

67. Cheng K, et al. Magnetic enhancement of cell retention, engraftment, and functional benefit after intracoronary delivery of cardiac-derived stem cells in a rat model of ischemia/reperfusion. Cell Transplant. 2012;21(6):1121-1135.

68. de Couto G, Kanazawa H, Tseliou E, Marbán L, Marbán E. Abstract 15884: Cardiospherederived cells confer acute cardioprotection following ischemia-reperfusion injury in rats: role of macrophage polarization. Circulation. 2014:130:A15884.

69. Nahrendorf M, et al. Dual channel optical tomographic imaging of leukocyte recruitment and protease activity in the healing myocardial infarct. Circ Res. 2007;100(8):1218-1225.
70. Chow A, et al. Bone marrow CD169+ macrophages promote the retention of hematopoietic stem and progenitor cells in the mesenchymal stem cell niche. J Exp Med. 2011;208(2):261-271.

71. Ehninger A, Trumpp A. The bone marrow stem cell niche grows up: mesenchymal stem cells and macrophages move in. J Exp Med. 2011;208(3):421-428.

72. Smith JN, Calvi LM. Concise review: Current concepts in bone marrow microenvironmental regulation of hematopoietic stem and progenitor cells. Stem Cells. 2013;31(6):1044-1050.

73. Winkler IG, et al. Bone marrow macrophages maintain hematopoietic stem cell (HSC) niches and their depletion mobilizes HSCs. Blood. 2010;116(23):4815-4828.

74. Godwin JW, Pinto AR, Rosenthal NA. Macrophages are required for adult salamander limb regeneration. Proc Natl Acad Sci U S A. 2013;110(23):9415-9420.

75. Zhang C, Li Y, Wu Y, Wang L, Wang X, Du J. Interleukin-6/signal transducer and activator of transcription 3 (STAT3) pathway is essential for macrophage infiltration and myoblast proliferation during muscle regeneration. J Biol Chem. 2013;288(3):1489-1499.

76. Reimer KA, Lowe JE, Rasmussen MM, Jennings RB. The wavefront phenomenon of ischemic cell death. 1. Myocardial infarct size vs duration of coronary occlusion in dogs. Circulation. 1977;56(5):786-794.

77. Sekar RB, et al. IK1 heterogeneity affects genesis and stability of spiral waves in cardiac myocyte monolayers. Circ Res. 2009;104(3):355-364. 